Monatsschr Kinderheilkd 2015 · 163:1269-1286 DOI 10.1007/s00112-015-0003-9

c) Die Autoren 2015

\section{Redaktion}

A. Borkhardt, Düsseldorf

S. Wirth, Wuppertal

CrossMark

\section{J. Pfeil $\cdot$ R. Kobbe ${ }^{2} \cdot$ S. Trapp ${ }^{3} \cdot$ C. Kitz ${ }^{4} \cdot$ M. Hufnagel ${ }^{5}$}

${ }^{1}$ Kinderheilkunde I, Zentrum für Kinder- und Jugendmedizin, Universitätsklinikum Heidelberg, Heidelberg, Deutschland

${ }^{2}$ Klinik und Poliklinik für Kinder- und Jugendmedizin, Universitätsklinikum Hamburg-Eppendorf, Hamburg, Deutschland

${ }^{3}$ Bremen, Deutschland

${ }^{4}$ Kinder- und Jugendmedizin, Missionsärztliche Klinik, Würzburg, Deutschland

${ }^{5}$ Sektion Pädiatrische Infektiologie und Rheumatologie, Klinik I, Zentrum für Kinder- und Jugendmedizin, Universitätsklinikum Freiburg, Freiburg, Deutschland

\title{
Empfehlungen zur infektiologischen Versorgung von Flüchtlingen im Kindes- und Jugendalter in Deutschland
}

\author{
Stellungnahme der Deutschen Gesellschaft \\ für Pädiatrische Infektiologie, der \\ Gesellschaft für Tropenpädiatrie und \\ Internationale Kindergesundheit und des \\ Berufsverbandes der Kinder- und \\ Jugendärzte
}

\section{Hintergrund}

Deutschland und Europa erleben momentan eine zahlenmäßig unabsehbare Zuwanderung von Flüchtlingen aus dem Nahen und Mittleren Osten (v. a. Syrien, Afghanistan, Irak und Pakistan), den afrikanischen Staaten (v. a. Eritrea, Nigeria), sowie dem westlichen Balkan (v. a. Albanien, Bosnien-Herzegowina, Kosovo, Mazedonien und Serbien - bei zuletzt abnehmenden Zahlen) und der Ukraine [5]. Mindestens jeder vierte Asylbewerber ist ein Kind oder Jugendlicher [9]. Bürgerkriege, Katastrophen und zunehmende Armut zwingen viele Menschen zur entbehrungsreichen Flucht in die Länder West- und Nord-Europas. Hieraus resultiert auch ein relevant erhöhter medizinischer Bedarf bei der medizinischen Versorgung vieler Flüchtlinge in Deutschland.
Die Grundhaltung zur ärztlichen Versorgung von minderjährigen Flüchtlingen leitet sich aus dem Artikel 24 [Gesundheitsvorsorge] der UN-Kinderrechtskonvention von 1989 her. Die Vertragsstaaten erkennen das Recht des Kindes auf das erreichbare Höchstmaß an Gesundheit an. Flüchtlinge sind auf dem gleichen medizinischen Niveau zu versorgen wie die einheimische Wohnbevölkerung. Dies gilt für akute oder chronische Erkrankungen ebenso wie für alle Maßnahmen der medizinischen Prävention. In der aktuellen Ausnahmesituation mit dem plötzlichen massenhaften Auftreten von Leistungssuchenden können die Versorgungskapazitäten überfordert sein. Mögliche Versorgungslücken sollten dann in der weiteren medizinischen Betreuung geschlossen werden.

Eine aktuelle Querschnittserhebung unter 100 syrischen Flüchtlingskindern in einer Münchner Erstaufnahmestelle ergab, dass $80 \%$ der Kinder unter somatischen (v. a. Atemwegserkrankungen) und $40 \%$ unter psychischen/psychiatrischen Beschwerden (v. a. posttraumatische Belastungsstörungen) litten. Unter den somatischen Beschwerden fanden sich in $10 \%$ infektiöse/parasitäre Erkrankungen (in erster Linie Hautinfektionen), weiterhin wiesen $40 \%$ der Kinder, die Impfdokumente hatten, einen unvollständigen Impfstatus auf [7]. Häufig sind die Informationen zur medizinischen Anamnese und zum Impfstatus aufgrund der Sprachbarriere schwer zu erheben, fragmentarisch oder sie fehlen ganz.

Flüchtlinge und Asylsuchende sind eine besonders vulnerable Gruppe in unserer Gesellschaft. Dies gilt insbesondere für Kinder und Jugendliche, die teilweise auch unbegleitet kommen, und für Schwangere. Sie benötigen eine angemessene und niederschwellige medizinische Versorgung, die an die individuelle Si- 


\section{Information \\ Diese Stellungnahme wurde von den beteiligten Autoren im Auftrag der jeweiligen Fachgesellschaften erarbeitet: \\ Für die Deutsche Gesellschaft für Pädiatrische Infektiologie (DGPI e. V): Dr. Johannes Pfeil, Heidelberg PD Dr. Markus Hufnagel, Freiburg}

Für die Gesellschaft für Tropenpädiatrie und Internationale Kindergesundheit (GTP e. V.): PD Dr. Robin Kobbe, Hamburg

Dr. Christa Kitz, Würzburg

Für den Berufsverband der Kinder- und Jugendärzte (BVKJ e. V.):

Dr. Stefan Trapp, Bremen

\section{Mitarbeit}

An der Erarbeitung der Stellungnahme waren Vertreter der folgenden Fachgesellschaften und Organisationen beteiligt:

- Deutsche Akademie für Kinder- und Jugendmedizin, Kommission für Infektionskrankheiten und Impffragen (DAKJ),

- Deutsche Gesellschaft für Gynäkologie und Geburtshilfe (DGGG),

- Deutsche Gesellschaft für Kinder- und Jugendmedizin (DGKJ),

- Deutsche Gesellschaft für Tropenmedizin und Internationale Gesundheit (DTG),

Folgende Personen haben an der Erarbeitung der Stellungnahme mitgewirkt:

- Prof. Dr. Ralf Bialek (Infektionsdiagnostik; DGPI),

- Prof. Ulrich Heininger (Impfungen; Sprecher der Kommission für Infektionskrankheiten und Impffragen der DAKJ, DGPI),

- Prof. Dr. Hans-lko Huppertz (Gesamtkonzept; DAKJ, DGPI),

- Prof. Dr. Thomas Junghanss (Tropenmedizinische Aspekte; DTG),

- Dr. Carsten Krüger (Gesamtkonzept; GTP),

- Dr. Mirjam Kunze (Schwangerenvorsorge; DGGG),

- Prof. Dr. Johannes Liese (Gesamtkonzept, MRE-Screening; DGPI, DGKJ),

- PD Dr. Nicole Ritz, PhD (Tuberkulose; DGPI - Ausschuss typische und atypische Mykobakteriosen),

- PD Dr. Erika Sievers, MPH (Aspekte öffentlicher Kinder- und Jugendgesundheitsdienst; Fachausschuss Kinder- und Jugendgesundheitsdienst),

- Prof. Dr. Arne Simon (MRE-Screening; DGPI),

- Prof. Dr. August Stich (Gesamtkonzept tropenmedizinische Aspekte; DTG). tuation angepasst sein muss. Es ist eine professionelle, soziale und ethische Herausforderung, die medizinische Versorgung von Flüchtlingen adäquat zu organisieren und durchzuführen.

Infektiologische Fragen oder Beschwerden nehmen einen besonderen Stellenwert bei der Betreuung von Flüchtlingen ein [7, 15]. Die vorliegende Empfehlung konzentriert sich bewusst auf die Infektionsdiagnostik und Infektionsprävention, wohl wissend, dass Kindergesundheit weit darüber hinausgeht. Weitere Handlungsempfehlungen von Behörden und anderen Fachgesellschaften, die sich mit anderen gesundheitsbezogenen Aspekten und der psychosozialen Betreuung von Kindern und Jugendlichen befassen, die als Flüchtlinge nach Deutschland kommen, sind bereits publiziert $[1,15,19]$ oder werden folgen.

Die vorliegenden Empfehlungen sollen Ärzte und medizinisches Personal in der medizinischen Versorgung von Flüchtlingen im Kindes- und Jugendalter unterstützen mit dem Ziel,

1. einen unvollständigen Impfschutz frühzeitig zu erkennen und rasch $\mathrm{zu}$ vervollständigen - zum individuellen Schutz und um Ausbreitungen von Infektionen zu verhindern,

2. übliche Infektionskrankheiten im Kindes- und Jugendalter, auch vor dem Hintergrund von Sammelunterkünften, Sprachbarrieren und unterschiedlichen kulturellen Auffassungen, zu diagnostizieren und $\mathrm{zu}$ behandeln und

3. in Deutschland seltene Infektionskrankheiten (z. B. Tuberkulose, Malaria, Dengue-Fieber, kutane Leishmaniose) frühzeitig zu erkennen und zu therapieren.

Der Bundesverband der Kinder- und Jugendärzte (BVKJ), die Deutsche Gesellschaft für Pädiatrische Infektiologie (DGPI) und die Gesellschaft für Tropenpädiatrie und Internationale Kindergesundheit (GTP) wollen mit dieser Stellungnahme Hilfestellung leisten, welche RoutineMaßnahmen in der Infektionsdiagnostik und Infektionsprävention bei der medizinischen Versorgung von Flüchtlingen im Kindes- und Jugendalter sinnvoll sind.
Die Stellungnahme beinhaltet keine Empfehlungen zum individuellen Umgang akuter infektiologischer Probleme. Hierzu wird auf Empfehlungen und Leitlinien der Fachgesellschaften (z. B. DGPI-Handbuch - Infektionen bei Kindern und Jugendlichen [6] oder RKI - Infektionskrankheiten von A-Z [17]) verwiesen. Das Robert Koch-Institut hat in einer aktuellen Publikation eine Liste mit den häufigsten ungewöhnlichen Infektionskrankheiten zusammengefasst, die bei der aktuellen Flüchtlingspopulation zu erwarten sind [15]. In - Tab. 1 sind die häufigsten Leitsymptome, ihre wichtigsten infektiologischen Differenzialdiagnosen und eine Basisdiagnostik tabellarisch zusammengefasst. Sollte die Basisdiagnostik keinen wegweisenden Befund erbringen, ist die Kontaktaufnahme zu einem pädiatrischen Infektiologen/einer pädiatrischen Infektiologin oder einem Tropenmediziner/einer Tropenmedizinerin empfohlen.

Wichtig ist jedoch, $\mathrm{zu}$ betonen, dass „klassische“ Infektionskrankheiten, z. B. akute respiratorische Infekte, MagenDarm-Infektionen und pyogene Hautund Weichteilinfektionen bei Flüchtlingen häufiger sind als die in unseren Breiten seltenen importierten Infektionskrankheiten. Ebenso wichtig ist die Einschätzung, dass von Flüchtlingen weder für die Allgemeinbevölkerung noch für ihre Helfer ein generelles erhöhtes Infektionsrisiko ausgeht. Flüchtlinge sind aus infektiologischer Sicht keine gefährliche, sondern aufgrund der besonderen Lebensumstände eine gefährdete Gruppe. Den Mitarbeitern in den Gemeinschaftseinrichtungen für Flüchtlinge, auch den ehrenamtlich Tätigen sowie der ansässigen Bevölkerung, sollte in diesem Zusammenhang nochmals die Bedeutung von Impfungen kommuniziert und ihnen ein Impfschutz nach den Empfehlungen der STIKO - wann immer möglich - angeboten werden.

Diese Stellungnahme basiert auf praktischen Erfahrungen von Experten der pädiatrischen Infektiologie und der internationalen Gesundheit im Kindesund Jugendalter in Deutschland. Frühere Empfehlungen der Kommission für Infektionskrankheiten und Impffragen der Deutschen Akademie für 
Hier steht eine Anzeige.

算 Springer 
Monatsschr Kinderheilkd 2015 • 163:1269-1286 DOI 10.1007/s00112-015-0003-9

(c) Die Autoren 2015

J. Pfeil · R. Kobbe ·S. Trapp · C. Kitz · M. Hufnagel

Empfehlungen zur infektiologischen Versorgung von Flüchtlingen im Kindes- und Jugendalter in Deutschland. Stellungnahme der Deutschen Gesellschaft für Pädiatrische Infektiologie, der Gesellschaft für Tropenpädiatrie und Internationale Kindergesundheit und des Berufsverbandes der Kinder- und Jugendärzte

\section{Zusammenfassung}

Minderjährige Flüchtlinge in Deutschland bilden eine besonders vulnerable Gruppe in unserer Gesellschaft. Dabei spielen Infektionskrankheiten eine wesentliche Rolle bei der medizinischen Versorgung. Aus infektiologischer Sicht sind Flüchtlinge keine gefährliche, sondern aufgrund der besonderen Lebensumstände eine gefährdete Gruppe. Auch in Krisensituationen erfordern ethische und ärztliche Verpflichtungen, ein Höchstmaß an medizinischer Versorgung zu erreichen. Die hier dargestellten Empfehlungen zur Infektionsdiagnostik und -prävention von Flüchtlingen im Kindes- und Jugendalter dienen dazu, den Impfschutz zu optimieren und Infektionskrankheiten, auch vor dem Hintergrund von Sammelunterkünften,
Sprachbarrieren und unterschiedlichen kulturellen Auffassungen, zu diagnostizieren, zu behandeln und deren Weiterverbreitung zu verhindern.

In den Erstaufnahmestellen sollen durch ein Kurzscreening (besser durch eine frühzeitige Basisuntersuchung) akute medizinische Probleme, potenziell übertragbare Infektionen (inkl. Tuberkulose), spezifische Impflücken, aber auch andere behandlungsbedürftige Erkrankungen erkannt und behandelt werden. Die Dokumentation aller Befunde ist essenziell, um Doppeluntersuchungen zu vermeiden und die weitere Behandlung zu optimieren. Hierfür ist eine funktionierende Kommunikationsstruktur zu schaffen.
Nach Verteilung der Flüchtlinge auf die Kommunen sollen im Rahmen der ambulanten und evtl. stationären Versorgung die von der STIKO empfohlenen Standardimpfungen vervollständigt und Flüchtlinge in allen medizinischen Bereichen mit dem gleichen medizinischen Niveau versorgt werden wie die einheimische Bevölkerung. Wegen einer höheren Prävalenz von multiresistenten Erregern (MRE) in den Herkunftsländern ist bei stationären Aufnahmen in vielen Fällen ein MRE-Screening empfohlen.

Schlüsselwörter

Übertragbare Krankheiten · Stationär behandelte Patienten . Prävention und Kontrolle $\cdot$ Tuberkulose $\cdot$ Impfungen

\section{Recommendations for the diagnosis and prevention of infectious diseases in pediatric and adolescent refugees in Germany. Statement of the German Society of Pediatric Infectious Diseases, the Society of Tropical Pediatrics and International Child Health, and the Professional Association of Pediatricians}

\begin{abstract}
Child and adolescent refugees in Germany represent a particularly vulnerable social group and treating infectious diseases forms a crucial part of providing their medical care. From an infectious diseases perspective, refugees themselves, as a result of their difficult personal circumstances, are the ones at highest risk. Even in crisis situations, medical practitioners are medically and ethically obliged to provide a high standard of care. The guidelines presented here propose recommendations for diagnosing and preventing infectious diseases among refugees under 18 in Germany. The guidelines are intended to assist in optimizing vaccine protection and treatment of diseases while taking into consideration factors such as
\end{abstract}

refugees' challenging living conditions, cultural differences and potential language barriers.

Upon refugees' arrival at the first housing sites, it is recommended that a basic clinical screening (and not just a brief visual inspection) be provided in order to identify and initiate treatment for acute medical problems and potentially contagious diseases (including tuberculosis), as well as to close gaps in vaccination coverage. Documentation of the clinical findings is critical, both to avoid redundant investigations and to optimize individual medical care. For this, an effective communication system must be established. Once refugees have been transferred into their destination community, outpatient and inpatient care providers should collaborate to bring refugees up-to-date with all vaccines recommended by STIKO (German Standing Committee on Vaccination). The same high standard of medical care should be delivered to refugees as would be to the general population. Due to the high prevalence of multi-resistant organisms (MRO) in the refugees' countries of origin, MRO screening is recommended for most patients receiving inpatient care.

\section{Keywords}

Communicable diseases - Inpatients . Prevention \& control - Tuberculosis . Vaccination
Kinder- und Jugendmedizin (DAKJ; zuletzt aktualisiert im Jahr 2013 [19]) bzw. der American Academy for Pediatrics (zuletzt aktualisiert im Jahr 2012 [1]) und der US-amerikanischen Centers for Disease Control and Prevention (vom August 2012 [25]) wurden in der Erstellung zum Teil berücksichtigt.
Die Empfehlungen der DAKJ [19] unterscheiden sich von dieser Stellungnahme, da zum einen die umfassende medizinische Versorgung (nicht fokussiert auf infektiologische Aspekte) abgehandelt ist und die Empfehlungen für ,einzelne“ Immigranten/internationale Adoptivkinder gedacht sind. Die große Anzahl der Flüchtlinge in Deutschland zwingt neben der „bestmöglichen“ Versorgung des Einzelnen, zusätzlich Kosten- und Praktikabilitätsaspekte zu berücksichtigen. 
Tab. 1 Symptom- und befundorientierte regionenspezifische infektiologische/tropenmedizinische Differentialdiagnosen bei Flüchtlingen im Kindes- und Jugendalter

Leitsymptom/Leitbefund Infektiologische/tropenmedizinische Erstdiagnostik bzw. Differentialdiagnostik

\begin{tabular}{|ll|}
\hline $\begin{array}{l}\text { Akute Dysenterie (blutiger Stuhl, } \\
\text { Fieber, Bauchschmerzen) }\end{array}$ & $\begin{array}{l}\text { Stuhluntersuchung auf pathogene Bakterien (Kultur) auf enteroinvasive bakterielle Infektionen }(A-E) \\
\text { Stuhluntersuchung auf Amoeben (Entamoeba histolytica) und Amoeben-Serologie bei V. a. Amoeben-Co- } \\
\text { litis }(A-E)\end{array}$ \\
\hline $\begin{array}{l}\text { Chronische Diarrhoe } \\
\text { (> 14 Tage) }\end{array}$ & $\begin{array}{l}\text { Stuhluntersuchung auf Giardia lamblia (PCR/Antigen/Mikroskopie) }(D-E) \\
\text { Serologie auf HIV, insbesondere } D, E \\
\text { häufige DD: Laktoseintoleranz (inbesondere } B, C, E)\end{array}$ \\
\hline
\end{tabular}

Periodisches Fieber und Bauchschmer- Überweisung an Zentrum mit Fragestellung Familiäres Mittelmeerfieber (insbesondere Mittelmeeranraizen mit Erhöhung von Leukozyten, CRP nerstaaten) und BSG

\begin{tabular}{|l|}
\hline Fieber und Raumforderung Leber \\
\hline $\begin{array}{l}\text { Zystische Raumforderung insbesonde- } \\
\text { re Leber und/oder Lunge }\end{array}$ \\
\hline $\begin{array}{l}\text { Gedeihstörung, Pulmonale Sympto- } \\
\text { matik, pathologische Lymphknoten, } \\
\text { Aszites, Pleura-, Perikarderguss und } \\
\text { weitere Organmanifestationen extra- } \\
\text { pulmonaler Tuberkulose }\end{array}$ \\
\hline
\end{tabular}

Fieber ohne klinischen Fokus

Blutkultur

Amoebenserologie (DD Amoeben-Leberabszess; $A-E$ ) - Vorsicht: bei Patienten aus Endemiegebieten kann eine positive Amoeben-Serologie auch eine serologische Narbe einer früheren Infektion darstellen

Überweisung an Zentrum mit Fragestellung zystische Echinokokkose $(A-E)$

(Online-Anfrage über www.tropenmedizin-heidelberg.de- „Konsiliaranfrage Echinokokkose“)

THT und/oder IGRA, Bildgebung und mykobakterielle Diagnostik: pulmonale und extrapulmonale Tuberkulose $(A-E)$

Serologie auf HIV (insbesondere $D, E$ )

\begin{tabular}{|ll}
\hline Fieber ohne klinischen Fokus & $\begin{array}{l}\text { Dicker Tropfen und dünner Blutausstrich, ggfls. ergänzend Schnelltest bis } 1 \text { Jahr nach Ankunft in Deutsch- } \\
\text { land bei Malaria (Verdacht bereits medizinischer NOTFALL) }(C, E)\end{array}$ \\
& $\begin{array}{l}\text { Blutkultur (u. a. Salmonella typhi) bei u. a. Typhus abdominalis }(A-E) \\
\text { Leishmanien-Antikörper (insbesondere bei Hepatosplenomegalie und Panzytopenie) bei viszeraler Leish- } \\
\text { maniasis ( } A-E \text { ) }\end{array}$ \\
\hline Zerebraler Krampfanfall & Bildgebung (DD Neurozystizerkose; $A-E$ ) \\
\hline Eosinophilie (> 500/nl) & $\begin{array}{l}\text { Stuhl auf Wurmeier (3 Stuhlproben von verschiedenen Tagen) auf intestinale Helminthen (Wurmeier im } \\
\text { Stuhl sind oft erst verzögert nachweisbar, da Eosinophilie erst während der Gewebspassage ausgeprägt } \\
\text { ist) } \\
\text { Strongyloides-Serologie bzw. Strongyloides-PCR im Stuhl } \\
\text { Falls negativ, } \\
\text { umfangreiches Gewebshelminthen-Screening in Absprache mit pädiatrisch-infektiologischem/ } \\
\text { tropenmedizinischem Zentrum }\end{array}$ \\
\hline Transaminasenerhöhung & Serologie auf Hepatitis A, B, C und E, EBV, CMV (A-E) \\
\hline $\begin{array}{l}\text { Splenomegalie, ultrasonographische } \\
\text { Zeichen einer Leberfibrose, Zeichen der } \\
\text { portalen Hypertension }\end{array}$ & $\begin{array}{l}\text { Überweisung an pädiatrisch-infektiologisches/tropenmedizinisches Zentrum (DD gastrointestinale } \\
\text { Schistosomiasis, insbesondere } E \text { ) }\end{array}$ \\
\hline
\end{tabular}

Rezidivierende Harnwegsinfekte, Überweisung an pädiatrisch-infektiologisches/tropenmedizinisches Zentrum (DD urogenitale Schistosoultrasonographische Zeichen von Blasenwandveränderungen, Harnabflussstörungen

\begin{tabular}{|ll|}
\hline Unklare Hautläsion - mit Juckreiz & $\begin{array}{l}\text { Frage nach nächtlichem Juckreiz, Hautinspektion auf Kratzspuren und skabies-typische Prädilektionsstel- } \\
\text { len (intertriginös, Genitalbereich) zur DD Skabies }\end{array}$ \\
\hline Unklare (chronische) Hautulzera & $\begin{array}{l}\text { Überweisung an pädiatrisch-infektiologisches/tropenmedizinisches Zentrum (DD kutane Leishmaniose, } \\
\text { insbesondere } B, C)\end{array}$ \\
\hline
\end{tabular}

Die Tabelle gibt nur eine Orientierung über wichtige regionenspezifische Differenzialdiagnosen häufiger Leitsymptome und -befunde. Die Auswahl der Regionen orientiert sich an der derzeitigen Häufigkeitsverteilung der Flüchtlingspopulationen in Deutschland:

A Westlicher Balkan, B Syrien, Irak, C Pakistan, Afghanistan, D Russische Föderation, Georgien, E Afrika südlich der Sahara.

Wird eine regionenspezifische infektiologische/tropenspezifische Verdachtsdiagnose gestellt, sollte sofort mit einem pädiatrisch-infektiologischen/ tropenmedizinischen Zentrum Kontakt aufgenommen werden - je nach Akutheit und insbesondere bei Verdacht auf Malaria am gleichen Tag. Wird keine Verdachtsdiagnose gestellt und persistieren die Symptome und Befunde, die zur Abklärung geführt haben, ist ebenfalls eine Kontaktaufnahme mit einem Zentrum für pädiatrische Infektiologie/Tropenmedizin empfohlen. 


\section{Gesundheitssystem und gesetzliche Vorschriften}

Kontakt zum deutschen Gesundheitssystem haben Flüchtlinge auf verschiedene Arten.

\section{Kurzscreening in den Erstaufnah- mestellen}

Ziel des Kurzscreenings (der „Inaugenscheinnahme") ist

1. die Früherkennung von potenziell übertragbaren Erkrankungen und

2. die Entscheidung, ob der neu angekommene Flüchtling in der Erstaufnahmestelle verbleiben kann oder sich akut in medizinische Behandlung (ambulant oder stationär) begeben muss.

Eine „Inaugenscheinnahme“ findet unmittelbar nach Ankunft der Flüchtlinge in der Erstaufnahmestelle statt. Hier spielen Durchfallerkrankungen, exanthematöse Erkrankungen und andere Erkrankungen mit hohem Übertragungsrisiko (insbesondere die Tuberkulose) eine entscheidende Rolle.

Die Tuberkulose ist in den Herkunftsländern der Flüchtlinge häufiger als in Deutschland. Flucht, unzureichende medizinische Versorgung und beengte sowie unhygienische Lebensbedingungen bergen ein zusätzliches Risiko, eine Tuberkulose zu erwerben oder zu reaktivieren. Gemäß $\$ 36$, Abs. 4 des Infektionsschutzgesetzes (IfSG) soll vor oder unverzüglich nach Aufnahme in eine Gemeinschaftsunterkunft/ Erstaufnahmeeinrichtung für Flüchtlinge oder Asylbewerber das Vorliegen einer ansteckungsfähigen Lungentuberkulose ausgeschlossen werden. Dafür sieht der Gesetzgeber die Ausstellung einer medizinischen Bescheinigung vor. Gesetzliche Vorgaben, wie dieser Ausschluss zu führen ist, existieren nur für Erwachsene und Jugendliche ab 15 Jahre. Für diesen Personenkreis muss sich die medizinische Bescheinigung (mit Ausnahme von Schwangeren) auf eine Röntgenaufnahme der Lunge stützen. Für alle asylsuchenden Kinder und Jugendliche $<15$ Jahren empfiehlt die Arbeitsgruppe „AWMF-Leitlinie Dia- gnostik, Prävention und Therapie der Tuberkulose im Kindes- und Jugendalter" ein immundiagnostisches TuberkuloseScreening mittels Tuberkulin-Hauttest (THT) oder Interferon-gamma Release Assay (IGRA) [2]. Bei positivem Testergebnis sollen weitere Abklärungen und eine Therapie gemäß bestehenden nationalen Empfehlungen erfolgen [6].

Die praktische Umsetzbarkeit eines solchen allgemeinen Tuberkulosescreenings erfordert personelle und logistische Unterstützung für die vor Ort Tätigen durch Politik und Geldgeber. Ohne eine solche Unterstützung wird ein flächendeckendes, zeitnahes Screening nicht möglich sein. Für die infektionshygienische Überwachung ist nach $₫ 36$ (1) IfSG der Öffentliche Gesundheitsdienst (ÖGD) verantwortlich.

Der Umfang des Kurzscreenings ist gesetzlich nicht vorgegeben.

Die Autoren dieser Stellungnahme unterstützen das Kölner Statement zur medizinischen Versorgung von Flüchtlingen vom 23.9.2015 [11], in dem es heißt, dass die reine (ärztliche) ,Inaugenscheinnahme" - neben einer möglichen Traumatisierung des Flüchtlings - eine Vergeudung von wertvollen Ressourcen ist, da für die Identifikation von vermeintlichen Ansteckungsgefahren eine strukturierte Anamnese und eine gezielte Untersuchung notwendig sind. Als bessere Alternative soll geschultes medizinisches Fachpersonal wie z. B. Gesundheits- und (Kinder-)Krankenpfleger und -pflegerinnen, medizinische Fachangestellte/Arzthelfer und Arzthelferinnen oder Sanitäter und Sanitäterinnen in ausreichender Zahl und mit ausreichendem Zeitdeputat in allen Erstaufnahmestellen als Ansprechpartner für alltägliche gesundheitliche Fragestellungen vorgehalten werden. Bei Notwendigkeit einer ärztlichen Expertise sollen die Flüchtlinge dann ärztlichem Personal vorgestellt werden. Idealerweise sollen Kurzscreening und Basisuntersuchung zusammengefasst werden.

Während die „Inaugenscheinnahme“ grundsätzlich zeitnah und ganztägig bei Ankunft der Personen erfolgen sollte, erfolgt die Basisuntersuchung zu festgelegten geordneten Sprechstundenzeiten im Tagesrhythmus der Einrichtung - mög- lichst innerhalb von 24 Stunden nach Ankunft in der Erstaufnahmestelle.

\section{Basisuntersuchung in den Erstaufnahmestellen}

Die Basisuntersuchung („Gesundheitscheck") sollte bei Flüchtlingen im Kindes- und Jugendalter idealerweise von einem Kinder- und Jugendarzt durchgeführt werden. Ziel dieser Untersuchung ist das Erkennen von

1. potenziell übertragbaren Erkrankungen und von

2. akut behandlungsbedürftigen, (auch) nicht infektiologischen Grunder-

krankungen.

Diese Untersuchung soll zeitnah nach der Erstaufnahme stattfinden, sie erfolgt ohne Behandlungsschein und wird von den Behörden, die für die Erstaufnahmestellen zuständig sind, organisiert und finanziert.

Der $₫ 62$ des Asylbewerberleistungsgesetz (AsylbLG) legt fest, dass zum Erkennen von kontagiösen Erkrankungen bestimmte Untersuchungen vom Flüchtling bzw. vom Asylbewerber/von der Asylbewerberin geduldet werden müssen. Hierzu können z. B. auch eine Blutentnahme, eine Stuhluntersuchung und ein Röntgenbild des Thorax gehören.

Der Umfang der Basisuntersuchung ist gesetzlich nicht vorgegeben.

Die Dokumentation der Befunde der Basisuntersuchung ist essenziell, um nachfolgenden medizinischen Einrichtungen die Informationen zur Verfügung zu stellen und Doppeluntersuchungen zu vermeiden! Ein Dokument über die Basisuntersuchung soll dem Flüchtling/dem Asylsuchenden mitgegeben werden, entweder in Papierform oder elektronisch (als digitales Medium könnten z. B. auch Fotoaufnahmen von Papierdokumenten mit den Smartphones der Flüchtlinge benutzt werden, in dessen Besitz viele Flüchtlinge sind). Ein entsprechendes Papierdokument ist z. B. in Bremen als „Bremer Gesundheitsheft" entwickelt worden ${ }^{1}$. Zusätzlich sollte eine elektro-

\footnotetext{
1 Das Dokument kann unter www.bvkj.de/ mitglieder/medien-und-materialien als pdf-Datei heruntergeladen werden.
} 


\begin{tabular}{|c|c|c|}
\hline $\begin{array}{l}\text { Kurzscreening / Basisuntersuchung } \\
\text { (in der Erstaufnahmestelle) }\end{array}$ & $\begin{array}{l}\text { Ambulante Versorgung } \\
\text { (in der Kommune) }\end{array}$ & Stationäre Versorgung \\
\hline $\begin{array}{l}\text { A1. Fokussierte Anamnese } \\
\text { - } \text { Aktuelle Beschwerden } \\
\text { - } \text { evamilienanamnese / Begleitpersonen } \\
\text { A2. Überprüfung Impfstatus } \\
\text { A3. Fokussierte Untersuchung } \\
\text { - Gewicht, Länge } \\
\text { - Haut, Lymphknoten } \\
\text { - Herz, Lunge, Abdomen } \\
\text { B. Empfohlene Impfungen } \\
\text { - Masern, Mumps, Röteln, Varizellen } \\
\text { - } 1 \text { Tetanus, Priorität) } \\
\text { Pertussis; saisonale Influenza } \\
\text { (2. Priorität) } \\
\text { - Rotaviren, Meningokokken C / ACWY } \\
\text { (3. Priorität) } \\
\text { - Pneumokokken, humane Papilloma- } \\
\text { viren (4. Priorität) } \\
\text { C. Tuberkulose-Screening } \\
\text { - THT (<5 Jahren 1. Wahl; 5-15 Jahre) } \\
\text { - Röntgen (5-15 Jahre) }\end{array}$ & $\begin{array}{l}\text { A1. Vollständige Anamnese } \\
\text { A2. Überprüfung Impfstatus } \\
\text { A3. Vollständige körperliche } \\
\text { Untersuchung } \\
\text { B. Blutentnahme } \\
\text { - Differentialblutbild } \\
\text { - Serologien für HIV, Hepatitis B (bei } \\
\text { Herkunft aus Hochprävalenzgebieten) } \\
\text { C. Empfohlene Impfungen } \\
\text { - Masern, Mumps, Röteln, Varizellen } \\
\text { - (1. Priorität) } \\
\text { Tetanus, Diphtherie, Poliomyelitis, } \\
\text { Pertussis; saisonale Influenza } \\
\text { (2. Priorität) } \\
\text { - Rotaviren, Meningokokken C / ACWY, } \\
\text { - Hepatitis A (3. Priorität) } \\
\text { Hepatitis B, H. influenzae Typ b, } \\
\text { Pneumokokken, humane Papilloma- } \\
\text { viren (4. Priorität) } \\
\text { D. Evtl. Tuberkulose-Screening } \\
\text { nachholen }\end{array}$ & $\begin{array}{l}\text { A1. Vollständige Anamnese } \\
\text { A2. Überprüfung Impfstatus } \\
\text { A3. Vollständige körperliche } \\
\text { Untersuchung } \\
\text { B. Blutentnahme } \\
\text { - Differentialblutbild } \\
\text { - Serologien für HIV, HBV } \\
\text { (bei Hochprävalenzländern) } \\
\text { - symptomorientiert } \\
\text { C. MRE-Screening bei } \\
\text { - Krankenhausaufenthalt } \\
\text { - bekannte Kolonisierung } \\
\text { chronische Wunden / Haut- } \\
\text { läsionen } \\
\text { - } \quad \text { Unterbringung in Gemein- } \\
\text { schaftseinrichtungen } \\
\text { D. Evtl. Tuberkulose- } \\
\text { Screening nachholen } \\
\text { E. Evtl. Nachholimpfungen }\end{array}$ \\
\hline
\end{tabular}

Abb. $1<$ Inhalte der infektiologischen Versorgung von Flüchtlingen im Kindes- und Jugendalter in Deutschland nische, zentrale Dokumentation und Übermittlung der Befunde angestrebt werden, auf die nachfolgende Untersuchungsstellen zurückgreifen können.

\section{Ambulante Vorstellung bei niedergelassenen Ärzten/ Ärztinnen}

Eine Vorstellung bei den niedergelassenen Kollegen und Kolleginnen, die Kinder und Jugendliche versorgen, kann zur Basisuntersuchung (falls diese nicht in den Erstaufnahmestellen geleistet wird) oder bei akuten Erkrankungen erfolgen. Im letzteren Fall sollten die Flüchtlinge je nach landesrechtlicher Regelung - im Besitz einer Gesundheitskarte oder eines Behandlungsscheins sein, der nach Einzelantrag vom für sie zuständigen Sozialamt oder der Regierungsbehörde ausgestellt wird. In manchen Bundesländern, wie z. B. Nordrhein-Westfalen, reicht ein von der Erstaufnahmestelle vergebener Behandlungsschein zur ambulanten Weiterbehandlung aus. In Ländern wie Bremen und Hamburg erhalten Flüchtlinge nach ihrer Registrierung eine Gesundheitskarte. Mit dieser fallen Einzelanträge
aufBehandlungsscheine weg und erleichtern die medizinische Versorgung (mit reduziertem Leistungsanspruch gemäß AsylbLG). Entsprechende Versichertenkarten sollten in allen Bundesländern ausgestellt werden.

\section{Stationäre Vorstellung in den Kinderkliniken bei akuten Erkrankungen}

Auch für diese Behandlung sollte eine Gesundheitskarte bzw. ein Behandlungsschein des zuständigen Sozialamtes oder der Regierungsbehörde vorliegen, über den die Übernahme der Behandlungskosten gesichert ist. Notfallbehandlungen sind immer ohne Behandlungsschein zu leisten.

\section{Praktisches Vorgehen}

BVKJ, DGPI und GTP schlagen folgendes Untersuchungsprogramm bei Flüchtlingen im Kindes- und Jugendalter nach Ankunft in Deutschland für die Erkennung und Prävention von Infektionskrankheiten vor (zusammenfassende Darstellung in • Abb. 1). Dabei sollte zur optimalen Nutzung ärztlicher Ressourcen das Kurzscreening mit der Basisuntersuchung kombiniert werden. Wichtig ist die Verfügbarkeit von Dolmetschern, wenn keine ausreichenden Deutschkenntnisse bei den Flüchtlingen vorhanden sind. Die Autoren der Stellungnahme befürworten eine Finanzierung von Dolmetschern über die gesetzlichen Krankenkassen.

\section{Basisuntersuchung in den Erstaufnahmestellen}

Ziel der Basisuntersuchung ist der Ausschluss von übertragbaren Infektionskrankheiten, akut behandlungsbedürftigen Erkrankungen und die Erhebung des Impfstatus. Das Untersuchungsprogramm umfasst Routineuntersuchungen, die obligat alle Flüchtlinge erhalten sollen, sowie spezielle Untersuchungen, die fakultativ bei bestimmten anamnestischen Hinweisen, Symptomen oder Befunden ergriffen werden sollen. Letztere sind unter Punkt „Ambulante Vorstellung“ dargestellt. 


\section{Empfohlene Routineunter- suchungen von Kindern und Flüchtlingen \\ Anamnese}

1. Aktuelle Beschwerden und Vorerkrankungen

- Hinweise auf übertragbare Erkrankungen, insbesondere exanthematöse Haut-, Durchfall- und Atemwegserkrankungen (inklusive Tuberkulose)

- Akut behandlungsbedürftige Erkrankungen

- Akut psychische Dekompensationsrisiken $^{2}$

2. Familienanamnese und Begleitpersonen

- Wer sind die Begleitpersonen? (Mit Dokumentation der Telefonnummer(n) der Begleitpersonen)

- Ansteckende Erkrankungen der Begleitpersonen (insbesondere Tuberkulose)

3. Überprüfen der vorhandenen Dokumentation bekannter TuberkulinHauttest-Ergebnisse oder Bluttests für Tuberkulose

\section{Erfragen des Impfstatus ${ }^{3}$}

- Nur Impfpässe oder Arztbriefe zählen als offizielle Impfdokumente ${ }^{4}$

\section{Klinische Untersuchung \\ - Gewicht, Länge \\ - Körperliche Untersuchung mit spezi- eller Beachtung von \\ - Haut: Hinweise auf Infektionen (u. a. Erkennen von Skabies, Läu- sebefall, Pyodermie, Masern, Varizellen), Verletzungen \\ - Lymphknoten (insbesondere tu- berkulöse Lymphadenitis als häu-}

\footnotetext{
2 Ein Screening auf posttraumatische Belastungsstörungen (engl. posttraumatic stress disorder, PTSD) ist prinzipiell sinnvoll, sollte jedoch nur dann angeboten werden, wenn auch eine psychologische Nachbetreuung im Fall eines positiven Ergebnisses gewährleistet ist. Andernfalls wird eine erneute Traumatisierung durch die Befragung, die nicht adäquat behandelt werden kann, riskiert.

3 Infos über ausländische Impfpläne: http:// apps.who.int/immunization_monitoring/ globalsummary/schedules
}

figste extrapulmonale Manifestation)

- Herz, Lunge, Abdomen

Erklärungen bzw. Begründungen des Untersuchungsprogramms:

1. Grundlage für die Erkennung von behandlungsbedürftigen Infektionskrankheiten sind die Anamnese sowie die körperliche Untersuchung. Aus logistischen Überlegungen werden diese im Rahmen einer Basisuntersuchung knapper und fokussierter gehalten, mit dem Ziel, schwere sowie hoch ansteckende Infektionskrankheiten zu erkennen.

2. Die Impfanamnese stellt einen wichtigen Teil der Basisuntersuchung dar. Hierdurch können Impflücken sicher erkannt werden und die notwendigen Nachholimpfungen zeitnah veranlasst werden, was insbesondere vor dem Hintergrund von Sammelunterkünften mit hohem Infektionsrisiko wichtig ist.

3. Kontrovers diskutiert werden können die Notwendigkeit und der Umfang einer Blutentnahme im Rahmen der Basisuntersuchung. Gerade bei Kindern bedeutet die Blutentnahme einen hohen logistischen Aufwand. Demgegenüber ist die frühzeitige Erkennung mit Einleitung adäquater Therapie- und Präventionsmaßnahmen bei einigen Infektionskrankheiten nur durch

\footnotetext{
${ }^{4}$ In einer aktuellen Stellungnahme des Robert Koch-Instituts vom 5.10.2015 [16] können bei Flüchtlingen ausnahmsweise mündliche Angaben zu erfolgten Impfungen auch ohne Impfdokumente Berücksichtigung finden, sofern sie als glaubwürdig eingeschätzt werden. Eine solche Ausnahme kann bei Flüchtlingen aus Syrien gemacht werden, die vor 2010 geimpft worden sind. Syrien hatte vor dem Ausbruch des Bürgerkriegs ein effizientes Impfprogramm mit exzellenten Impfraten. Wenn die Eltern oder der/die Jugendliche glaubhaft versichern können, dass vor Ausbruch des Bürgerkriegs eine vollständige Immunisierung nach dem syrischen Impfplan erfolgte, kann auf die Durchführung von vielen Nachholimpfungen verzichtet werden (Ausnahmen: Varizellen und Meningokokken Gruppe C bzw. ACWY Konjugatimpfstoff, da diese in Syrien nicht im Impfprogramm enthalten sind). Bleiben Zweifel, gilt das empfohlene Vorgehen bei den Nachholimpfungen.
}

Laboruntersuchungen möglich. Beispielsweise ist die Prävalenz der Hepatitis B in den Ursprungsländern der Flüchtlinge deutlich höher als in Deutschland (Balkanstaaten und Naher Osten im Bereich von je 2-4 \%, in afrikanischen Ländern südlich der Sahara 5 bis $>8 \%$ [22]). Auf Hepatitis B wird in diesen Ländern während der Schwangerschaft üblicherweise nicht gescreent.

4. Die routinemäßige Durchführung eines Tuberkulose-Screenings ist aufgrund der Vielzahl von Flüchtlingen eine Herausforderung für die praktische Umsetzung. Begründet wird die medizinische Notwendigkeit eines routinemäßigen Screenings mit der erhöhten Prävalenz der Tuberkulose innerhalb von Risikopopulationen wie Flüchtlingen [2]. Zuverlässige Daten zur Prävalenz der Tuberkulose bei Flüchtlingen in Deutschland fehlen. Die Prävalenz dürfte aber mindestens so hoch liegen wie im jeweiligen Herkunftsland der Flüchtlinge. Herkunftsländer von Flüchtlingen mit sehr hoher Tuberkuloseinzidenz (d. h. > 100 pro 100.000) sind: Afghanistan, Eritrea, Nigeria, Pakistan und die Ukraine [24]. Allerdings ist bekannt, dass Prävalenzdaten aufgrund der vielfältigen Expositionsrisiken in Krisengebieten ohne ausreichende medizinische Versorgung und während der Flucht unzuverlässig sind. In einem systemischen Review, der die nationalen Prävalenzdaten mit jenen in Krisenregionen verglich, konnte gezeigt werden, dass die Raten in Krisenregionen 2- bis 20-fach erhöht sind [13]. In die Abwägung eines routinemäßigen Tuberkulosescreenings muss letztendlich auch mit einbezogen werden, dass das Risiko einer Tuberkuloseübertragung, das von Kindern ausgeht, deutlich geringer ist als bei Erwachsenen (auch wenn Ansteckungen durch Sputumnegative Kinder und durch Säuglinge beschrieben sind).

Falls ein routinemäßiges Tuberkulosescreening mit THT oder IGRA personell und organisatorisch nicht bei allen Kindern und Jugendlichen in den Erstaufnahmestellen 
umsetzbar ist, sollte das Screening zeitlich prioritär im Rahmen der Basisuntersuchung bei Vorliegen eines der folgenden Kriterien [10] durchgeführt werden: (1) bekannter TuberkuloseKontakt, (2a) Gewichtsstagnation oder Gewichtsverlust, (b) Husten $>2$ Wochen Dauer, (c) unerklärtes Fieber $>1$ Woche, (d) persistierende Müdigkeit, oder (3) Herkunft aus einem Land mit hoher Tuberkuloseinzidenz (> 100 pro 100.000 Bewohner). Das fehlende Tuberkulosescreening soll unter Angaben der Gründe dokumentiert und zeitnah bei allen Kindern und Jugendlichen nachgeholt werden.

5. Eine routinemäßige Röntgenuntersuchung des Thorax zum Ausschluss einer Lungentuberkulose, wie sie bei erwachsenen Flüchtlingen Standard ist, ist bei Kindern und Jugendlichen unter 15 Jahren aufgrund der niedrigen Sensitivität bei relevanter Strahlenbelastung abzulehnen. Bei Jugendlichen ab 15 Jahren ist nach IfSG eine Röntgen-ThoraxUntersuchung vorgeschrieben, um das Nichtvorliegen einer infektiösen Lungentuberkulose bescheinigen $\mathrm{zu}$ können [18].

Wichtig wäre zum Zeitpunkt der Basisuntersuchung bereits die Erkennung akuter und chronischer behandelbarer Erkrankungen. Zeitliche Limitationen und Kommunikationsschwierigkeiten im Rahmen der Basisuntersuchung erschweren oder verhindern einen solchen Anspruch. Die Diagnostik muss dann auf die ambulante Versorgung nach Verteilung der Flüchtlinge auf die Kommunen verschoben werden.

Da die Erfahrungen im Umgang mit einer großen Anzahl an Flüchtlingen bisher beschränkt sind, müssen die Empfehlungen dieser Stellungnahme im Verlauf an neue Erkenntnisse angepasst werden („work in progress").

\section{Empfohlene Impfungen in Erstaufnahmestellen}

Frühzeitige Impfungen der Flüchtlinge nach Ankunft in Deutschland sollen

1. den individuellen Schutz des Flüchtlings sicherstellen und
Hier steht eine Anzeige.

\section{Springer}


Tab. 2 Anamnese bei Flüchtlingen im Kindes- und Jugendalter

Unterlagen zu Schwangerschaft und Geburt (falls zugänglich; v. a. bei Kindern $<6$ Jahren)

- Mütterliche Risikofaktoren vor/während der Schwangerschaft (z. B. Hepatitis-B-, Hepatitis-C-, HIV-, Lues-Status, mütterliche Medikamente, Drogen, Alkohol, Nikotin)

- Schwangerschaftswoche, Geburtsmodus, Geburtsgewicht, Geburtslänge, Kopfumfang bei Geburt, APGAR-Werte

- Geburts- oder postnatale Komplikationen

- Ergebnisse des Neugeborenen-Stoffwechselscreenings bzw. -Hörscreenings

Perzentilenkurven inkl. Kopfumfang (internationale WHO-Kurven ${ }^{\text {a }}$ verwenden)

Vernachlässigung, Missbrauch, Gewaltanwendung ${ }^{b}$

Ernährung (v. a. Eisen, Kalzium, Vitamin D, lod)

Meilensteine der Entwicklung

Verhaltensauffälligkeiten

Labor- oder bildgebende Vorbefunde

Impfstatus ${ }^{c}$

Tuberkulin-Hauttest- oder IGRA-Ergebnisse

Vorerkrankungen c (v. a. Hinweise auf chronische Erkrankungen)

Allergien

Medikamenteneinnahme

Arztberichte

Familienanamnese

Umweltrisiken (z. B. Blei-, Nikotin-Exposition)

Institutionalisierung

Aktuelle Beschwerden ' (insbesondere Fragen nach Husten, Fieber, Gewichtsverlust, Nachtschweiß, Hauterscheinungen, Juckreiz, Durchfall, Erbrechen, akute Schmerzen)

- Tab. 2 und 3 sind als Orientierungshilfe gedacht, an welche anamnestischen und klinischen Befunde bei der medizinischen Versorgung von Flüchtlingen im Kindes- und Jugendalter gedacht werden soll.

a Zusätzlich internationale WHO-Kurven (www.who.int/childgrowth/en) verwenden.

${ }^{b}$ Ein Screening auf posttraumatische Belastungsstörungen (engl. posttraumatic stress disorder, PTSD) ist prinzipiell sinnvoll, sollte jedoch nur dann angeboten werden, wenn auch eine psychologische Nachbetreuung im Fall eines positiven Ergebnisses gewährleistet ist. Andernfalls wird eine erneute Traumatisierung durch die Befragung, die nicht adäquat behandelt werden kann, riskiert.

'Mindestanforderungen an Anamnese und Untersuchung im Rahmen einer Basisuntersuchung.

2. Ausbrüche impfpräventabler Infektionserkrankungen verhindern oder begrenzen.

Um zeitnah eine hohe Impfquote sicherzustellen, sind Impfangebote so früh als möglich nach Ankunft in den Räumlichkeiten der Aufnahmeeinrichtungen empfohlen (sogenannte Kohortenimpfung). Dazu sollen alle Flüchtlinge einen vollständigen Impfschutz bzw. eine Erstimpfung nach den aktuellen STIKO-Empfehlungen zur „Umsetzung frühzeitiger Impfungen bei Asylsuchenden nach Ankunft in Deutschland" aufweisen bzw. erhalten [16].

Im Fokus der Verhinderung von Ausbrüchen steht ein möglichst rascher Impfschutz gegen Masern, Mumps, Röteln und Varizellen. Unter Berücksichtigung von Kontraindikationen (z. B.
Schwangerschaft, schwere Immunsuppression) haben Lebendimpfungen gegen diese Viruserkrankungen bei Nichtgeimpften in den Gemeinschaftseinrichtungen die höchste Priorität ${ }^{5}$. Auf eine Varizellenimpfung kann verzichtet werden, wenn durch die Eltern eine

\footnotetext{
${ }^{5}$ Um Ausbrüche ausreichend sicher vermeiden zu können, ist eine einmalige MMR+V-Impfung zunächst ausreichend. Die 1. Impfdosis sollte im Alter von 9 Monaten (bis zu einem Alter von 6 Jahren) aufgrund eines möglicherweise höheren Fieberkrampfrisikos als getrennte MMR+V-Impfung verabreicht werden [14], kann aber aus medizinischen oder organisatorischen Gründen auch als MMRV-Vierfachimpfung verabreicht werden [16]. Die 2. MMRV-Impfung (bevorzugt als Vierfachimpfstoff) soll zu Beginn des 2. Lebensjahres [14] nachgeholt werden. Hinweis: Der Vierfachimpfstoff Priorix Tetra ${ }^{\circledR}$ ist nur bis zu einem Alter von 12 Jahren zugelassen.
}

Varizellenerkrankung in der Anamnese des Kindes glaubhaft angegeben wird.

Darüber hinaus sind in Gemeinschaftseinrichtungen Ausbrüche von invasiven Meningokokkenerkrankungen (wie Meningitis, Sepsis), Pertussis und Influenza gefürchtet, weshalb diese Impfungen zusätzlich zu den Standardimpfungen allen Flüchtlingen im Kindesund Jugendalter in Erstaufnahmeeinrichtungen angeboten werden sollen.

Die Standardimpfung gegen Rotaviren soll jungen Säuglingen STIKO-konform verabreicht werden. Dadurch kann das Risiko für Rotavirusausbrüche in den Einrichtungen reduziert werden.

Auch Hepatitis A kann prinzipiell zu Ausbrüchen in Gemeinschaftseinrichtungen führen, allerdings ist derzeit die Ausprägung des Risikos nicht bekannt, weshalb zum jetzigen Zeitpunkt eine routinemäßige Hepatitis-A-Impfung in Erstaufnahmestellen nicht empfohlen werden kann.

Aufgrund des Ausbreitungspotentials von Varizellen-Infektionen in Gemeinschaftseinrichtungen soll die erste Impfung - als MMR + V-Impfung - ab dem Alter von neun Lebensmonaten vorgezogen werden. Die 2. MMRV-Impfung sollte zu Beginn des 2. Lebensjahres gegeben werden, um einen optimalen Schutz vor Masern zu haben. Dabei muss ein Mindestabstand von 4-6 Wochen zur 1. MMRV-Impfung beachtet werden. Da die Impftiter bei einer so frühen Immunisierung niedriger ausfallen, ist eine 3. MMRV-Impfung zwischen dem Alter von 15 und 23 Monaten (mindestens 3 Monate Abstand zur 2. MMRV-Impfung) empfohlen.

Bei bestehendem fehlenden Impfschutz gegen Pertussis soll diese Impfung - je nach Alter des Flüchtlings und Impfstatus - mit Diphtherie, Tetanus, Poliomyelitis, ggf. auch mit Hepatitis B und $H$. influenzae Typ b kombiniert werden. Siehe dazu die aktuellen Impfempfehlungen der STIKO [14].

Bei der Rotavirusimpfung ist zu beachten, dass möglicherweise ein geringfügig erhöhtes Risiko für Darminvaginationen (ca. 1-2 Fälle pro 100.000 geimpfte Kinder) innerhalb der 1 . Woche nach der 1. Rotavirusimpfung besteht. Dieses Risiko nimmt mit dem Alter der Impflinge zu. 


\section{Tab. 3 Klinische Untersuchung bei Flüchtlingen im Kindes- und Jugendalter ${ }^{a}$}

Vitalzeichen ${ }^{\text {a }}$ Körpertemperatur, Herzfrequenz, Atemfrequenz, evtl. Blutdruck

Gewicht $^{a}$, Länge ${ }^{a}$, Kopfumfang (mit Anlegen einer Perzentilenkurve; internationale WHO-Kurven ${ }^{\text {b }}$ verwenden), BMI

Komplette körperliche Untersuchung mit spezieller Beachtung von:

- Haut ${ }^{\text {a }}$ : Hinweise auf Infektionen (u. a. Erkennen von Skabies, Läusebefall, Pyodermie, Masern, Varizellen), angeborene Hauterkrankungen, Verletzungen

- Lymphknoten

- Herz ${ }^{a}$, Lunge ${ }^{a}$, Abdomen $^{a}$

- Genitalien: Hinweise auf Infektionen, sexueller Missbrauch, Mutilationen

- Neurologie: Entwicklungsstand

- HNO- und Zahnstatus

- Tab. 2 und 3 sind als Orientierungshilfe gedacht, an welche anamnestischen und klinischen Befunde bei der medizinischen Versorgung von Flüchtlingen im Kindes- und Jugendalter gedacht werden soll.

${ }^{a}$ Mindestanforderungen an Anamnese und Untersuchung im Rahmen einer Basisuntersuchung. b Zusätzlich internationale WHO-Kurven (www.who.int/childgrowth/en) verwenden.

Daher empfiehlt die STIKO dringend, die Impfserie frühzeitig - spätestens bis zum Alter von 12 Wochen - zu beginnen und vorzugsweise bis zum Alter von 16 Wochen (Rotarix ${ }^{\oplus}$ ) bzw. von 20-22 Wochen (RotaTeq ${ }^{\oplus}$ ) abzuschließen. Die Impfserie muss für Rotarix auf jeden Fall bis zum Alter von 24 Wochen und für RotaTeq ${ }^{\circledast}$ bis zum Alter von 32 Wochen abgeschlossen sein. Die Sorgeberechtigten müssen über die klinischen Symptome einer Invagination aufgeklärt werden können (cave: Sprachbarriere) und auch kurzfristig eine medizinische Vorstellung gewährleistet sein, falls ein Säugling symptomatisch wird.

$\mathrm{Zu}$ den empfohlenen Impfungen von medizinischem Personal und (ehrenamtlichen) Helfern wird auf die Anlage 4 der STIKO-Empfehlung vom 5. Oktober 2015 verwiesen [16].

\section{Ambulante Vorstellung}

Ambulante Vorstellungen bei Ärzten und Ärztinnen, die Kinder und Jugendliche versorgen, können zur Basisuntersuchung oder bei behandlungsbedürftigen akuten oder chronischen Erkrankungen erfolgen. Im Erkrankungsfall muss das Untersuchungsprogramm symptomorientiert individuell bestimmt werden. Bei Verdacht auf eine importierte Infektionskrankheit oder bei positiven Screeningbefunden soll frühzeitig Kontakt mit einem pädiatrischen Infektiologen/ einer pädiatrischen Infektiologin oder einem Tropenmediziner/einer Tropenmedizinerin aufgenommen werden.

\section{Anamnese und klinischer Untersuchungsbefund}

Neben Anamnese und klinischer Untersuchung (siehe - Tab. 2 und 3) sollen ein Basislabor bei allen Flüchtlingen abgenommen werden und ein Tuberkulosescreening durchgeführt werden, wenn dieses noch nicht in den Erstaufnahmestellen gemacht wurde.

Zur Weitergabe bereits erhobener medizinischer Befunde ist eine Kommunikationsstruktur/-kultur zwischen der lokalen Erstaufnahmestelle und den lokalen Praxisärzten und -ärztinnen notwendig. Eine solche Struktur existiert derzeit nicht flächendeckend und sollte aufgebaut werden. Hierbei sollten - außer bei akuter Notwendigkeit - die Untersuchungen möglichst dort zugeordnet werden, wo die strukturellen Bedingungen für die erforderliche Compliance und mögliche Therapie angenommen werden kann. Dies ist eher erst in der aufnehmenden Kommune der Fall. Selbstverständlich kann aufgrund von Befunden aus der Anamnese und/oder der klinischen Untersuchung des Kindes/Jugendlichen eine Erweiterung dieses Basislabors medizinisch indiziert sein.

\section{Blutentnahme}

Als Basislaboruntersuchungen werden Blutbild und Differenzialblutbild bestimmt. Weitere routinemäßige Blutentnahmen werden nur bei Flüchtlingen aus bestimmten Hochprävalenzgebieten ${ }^{8}$ für ausgewählte Infektionskrankheiten empfohlen:
- Serologien auf HIV ${ }^{9}$ (idealerweise kombinierter Antigen-Antikörpertest),

- Hepatitis B (HBs-Antigen).

Erklärungen bzw. Begründungen des Untersuchungsprogramms:

1. Die Prävalenz einer Anämie, in erster Linie einer EisenmangelAnämie (aber auch Hämoglobinopathien), ist bei Flüchtlingen hoch $[1,25]$ und kann jedoch auch auf eine chronische Infektion (z. B. HIV, Tuberkulose) hinweisen. Über das Differenzialblutbild kann außerdem eine Eosinophilie erkannt werden, die auf eine parasitäre Infektionserkrankung hinweisen und eine weitere Abklärung erfordern kann.

2. Die Auswahl der serologischen Testungen beschränkt sich auf chronische Infektionserkrankungen, die auch bei asymptomatischen Patienten entweder eine Therapie (HIV, ggf. Hepatitis B) erfordern oder ein wesentliches Übertragungsrisiko (HIV, Hepatitis B) aufweisen. Eine Testung auf Hepatitis $\mathrm{C}$ wird derzeit nicht empfohlen, da bisher für die Pädiatrie keine allgemein akzeptierte Therapie existiert und keine spezifische Prophylaxe möglich ist.

Eine Untersuchung von Antikörpern gegen spezielle tropische Infektionserreger wird bei anamnestischen und klinischen Hinweisen regionenspezifisch neben anderen Nachweisverfahren durchgeführt, in der Regel jedoch durch Tropenmediziner und -medizinerinnen. Es wird in nächster Zeit noch $\mathrm{zu}$ diskutieren sein, ob nicht regionenspezifisch ein Screening z. B. bzgl. Schistosomiasis sinnvoll ist, da chronische Infektionen lange asymptomatisch (und ohne Eosinophilie) verlaufen und zu schweren, irreversiblen Schäden führen können.

\footnotetext{
8 Als Hochprävalenzländer gelten Länder mit einer Erkrankungsprävalenz von $\geq 1 \%$ für HIV (d. h. derzeit nur in Ländern in Sub-Sahara Afrika; nicht Naher und Mittlerer Osten, z. B. Syrien) [23] bzw. $\geq 8 \%$ für Hepatitis B (d. h. derzeit nur in Ländern in Sub-Sahara Afrika; nicht Naher und Mittlerer Osten, z. B. Syrien) [22].

${ }^{9}$ Ein informiertes Einverständnis wird benötigt
} 


\section{Bestehende Impflücken gegen Masern, Mumps, Pertussis, Röteln und Varizellen sollen bei der Erstuntersuchung erkannt und durch entsprechende Impfungen möglichst zeitnah geschlossen werden [16].}

1. Dabei haben der Impfschutz gegen Masern, Mumps, Röteln und Varizellen aus Sicht des Patienten und der Gemeinschaft die höchste Priorität. ${ }^{6}$

2. Danach folgt in der Dringlichkeit der Individualschutz gegen Tetanus sowie der Schutz vor Diphtherie, Poliomyelitis und Pertussis (der auch aus der Sicht der Gemeinschaft zu fordern ist, um Ausbrüche zu verhindern).

3. Die Impfung aller Flüchtlinge im Kindesund Jugendalter in Gemeinschaftseinrichtungen gegen Influenza, Meningokokken der Serogruppe $C^{7}$ und bei Säuglingen unter 3 Monaten gegen Rotaviren (Abschluss der Impfung vorzugsweise bis zum Alter von 16-22 Wochen) ist zur Vermeidung der Ausbreitung von Ausbrüchen sinnvoll.

4. Auch die Standardimpfungen gegen Pneumokokken und humane Papillomaviren sollen zeitnah verabreicht werden, möglichst bevor die Flüchtlinge auf die Kommunen verteilt werden.
${ }^{6}$ Um Ausbrüche ausreichend sicher vermeiden zu können, ist eine einmalige MMRV-Impfung zunächst ausreichend. Die 2. MMRV-Impfung kann zu einem späteren Zeitpunkt (d. h. zu Beginn des 2. Lebensjahres [14]) nachgeholt werden.

7 Statt der in Deutschland empfohlenen Standardimpfung gegen Meningokokken der SerogruppeC istbei Flüchtlingen aus den derzeitigen Regionen die Impfung mit einem tetravalenten MenACWY-Konjugatimpfstoff alternativ zu erwägen, da die Flüchtlinge aus Ländern mit höherer Prävalenz von Meningokokken der Serogruppen A, W, Y kommen, deshalb mit diesen Erregern besiedelt sein können (was zu Ausbrüchen führen kann) und möglicherweise zu einem späteren Zeitpunkt Reisen in ihre Heimatländer (als sogenannte „Visiting Friends and Relatives" (VFR)) unternehmen. Aufgrund des vergleichbaren Preises der MenACWY-Konjugatimpfstoffe mit den MenC-Impfstoffen fallen bei Verwendung von MenACWY keine wesentlichen Zusatzkosten an.
3. Eine routinemäßige Untersuchung von Urin oder Stuhl ist ohne entsprechende Symptomatik nicht sinnvoll [25]. Bevor ein Stuhlscreening allgemein empfohlen werden kann, sind regionenspezifische Daten zur Prävalenz von Darmparasitosen unter den Flüchtlingen im Kindes- und Jugendalter in Deutschland notwendig. Erst wenn diese Daten vorliegen, kann über die empirische Gabe von luminalen Antihelmintika (z. B. Mebendazol) als Alternative [20] zum Stuhlscreening oder gar keiner Maßnahme entschieden werden.

Eventuell muss ein immundiagnostisches Tuberkulosescreening mittels Tuberkulin-Hauttest (THT) oder Interferongamma Release Assay (IGRA) ${ }^{10}$ nachgeholt werden, falls ein solches nicht in der Erstaufnahmeeinrichtung stattgefunden hat.

Ein Tuberkulosescreening soll nach den Vorgaben der Arbeitsgruppe „AWMFLeitlinie Diagnostik, Prävention und Therapie der Tuberkulose im Kindesund Jugendalter" bei allen asylsuchenden Kindern und Jugendlichen $<15$ Jahren unabhängig von der Tuberkuloseinzidenz des Herkunftslandes durchgeführt werden [2]. Bei positivem Testergebnis sollen weitere Abklärungen und die Therapie gemäß bestehenden nationalen Empfehlungen erfolgen. Wenn die Infektion weniger als 8 Wochen zurückliegt oder eine Miliartuberkulose vorliegt, können beide Teste negativ sein.

- Wahl des Testverfahrens bei Kindern $<5$ Jahren:

In dieser Altersgruppe soll in erster Linie ein Tuberkulin-Hauttest (THT) angelegt werden.

Bei eingeschränkter Verfügbarkeit von PPD RT-23 kann auch bei Kindern $<5$ Jahren ein IGRA verwendet werden.

\footnotetext{
10 Der IGRA hat den Vorteil gegenüber dem THT, dass keine zweite Vorstellung zum Ablesen des Testergebnisses notwendig ist und keine falsch-positiven Screening-Ergebnisse bei Z. $\mathrm{n}$. BCG-Impfung erfasst werden. Dafür ist der Test teurer als der THT und die Test-Performance bei Kindern unter 5 Jahren ist nicht ausreichend evaluiert.
}

- Wahl des Testverfahrens bei Kindern und Jugendlichen $\geq 5-15$ Jahren: Bei Kindern ab 5 Jahren kann ein Tuberkulin-Hauttest (THT) oder ein Interferon-gamma Release Assay (IGRA) verwendet werden.

- Interpretation des THT:

Die Interpretation des THT-Ergebnisses muss unter Berücksichtigung des BCG-Impfstatus (evtl. falschpositiv) und des Ernährungs- bzw. Krankheitsstatus (falsch-negativ bei schwerer Malnutrition oder nach Masernerkrankung) erfolgen. Eine Induration von $\geq 10 \mathrm{~mm}$ ist eine Indikation zur weiteren Untersuchung unabhängig vom BCG-Impfstatus.

- Interaktion THT/IGRA und Maserninfektion/-impfung:

Eine Masernimpfung oder -infektion kann zu einer temporären Suppression der zellvermittelten Immunantwort und damit zu einem falsch-negativen THT- oder IGRATestergebnis führen [21]. Das immunodiagnostische Screening sollte deshalb zeitgleich oder vor einer MMR(V)-Impfung durchgeführt werden. Falls eine Masernimpfung kürzlich verabreicht wurde oder eine Masernerkrankung vorliegt, sollten 4-6 Wochen Abstand zur Masernimpfung/-infektion für die Testung eingehalten werden [21].

Bei gesicherter Exposition und initial negativem THT oder IGRA muss der Test nach 3 Monaten wiederholt werden. Bei Kindern $<5$ Jahren soll in dieser Situation bis zum wiederholten Test eine Chemoprophylaxe mit Isoniazid gegeben werden [6].

Bei folgenden anamnestischen Hinweisen, Symptomen oder Befunden muss eine weiterführende Diagnostik eingeleitet werden und die Patienten sollen frühzeitig an einen pädiatrischen Infektiologen/eine pädiatrische Infektiologin oder einen Tropenmediziner/eine Tropenmedizinerin überwiesen werden:

- Durchfall (v. a. wenn blutig-schleimig, mit hohem Fieber oder chronisch [d. h. > 2 Wochen bestehend]), - Splenomegalie,

- Verdacht auf sexuellen Missbrauch (u. a. zum Ausschluss einer sexuell übertragbaren Erkrankung; Ab- 
Tab. 4 Empfohlene Altersgruppe und Anzahl der Impfstoffdosen bzw. Impfsequenz der empfohlenen Nachholimpfungen für Flüchtlinge im Kindes- und Jugendalter [14]

\begin{tabular}{|c|c|c|}
\hline Impfung & $\begin{array}{l}\text { Empfohlene Alters- } \\
\text { gruppe }\end{array}$ & Anzahl Impfstoffdosen bzw. Impfsequenz \\
\hline MMR & $\begin{array}{l}\text { 1-17 Jahre } \\
\text { nach 1.1.1970 Gebore- } \\
\text { ne }\end{array}$ & $\begin{array}{l}0-1 \text { Monat } \\
\text { einmalig MMR }\end{array}$ \\
\hline Varizellen & 1-17 Jahre & 0-1 Monat \\
\hline Diphtherie & Immer & $0-1-6$ Monate \\
\hline Tetanus & Immer & 0-1-6 Monate \\
\hline Poliomyelitis & Immer & 0-1-6 Monate \\
\hline Pertussis & Immer & $\begin{array}{l}\text { Altersabhängig 1-4 Dosen (siehe STIKO-Empfeh- } \\
\text { lungen [14]) }\end{array}$ \\
\hline $\begin{array}{l}\text { H. influenzae } \\
\text { Typ b }\end{array}$ & $<5$ Jahre & $\begin{array}{l}\text { Altersabhängig 1-4 Dosen (siehe STIKO-Empfeh- } \\
\text { lungen [14]) }\end{array}$ \\
\hline Hepatitis $B^{a}$ & 0-17 Jahre & $\begin{array}{l}\text { 0-1-6 Monate; bei Impfungen von Säuglingen mit } \\
\text { 6-fachem Kombinationsimpfstoff sind } 4 \text { Impfdo- } \\
\text { sen notwendig }\end{array}$ \\
\hline Pneumokokken & $<2$ Jahre & $\begin{array}{l}\text { Alters- und gestationsaltersabhängig 1-4 Dosen } \\
\text { (siehe STIKO-Empfehlungen [14]) }\end{array}$ \\
\hline Rotavirus & $\begin{array}{l}\text { 6-12 Wochen }{ }^{\mathrm{b}} \text { (Beginn } \\
\text { der Impfserie) }\end{array}$ & $\begin{array}{l}2 \text { oder } 3 \text { Impfdosen (im 4-Wochen-Abstand; siehe } \\
\text { STIKO-Empfehlungen [14]) }\end{array}$ \\
\hline $\begin{array}{l}\text { Meningokokken } \\
\text { ACWY bzW. } C^{c}\end{array}$ & 1-17 Jahre & Einmalige Impfung \\
\hline $\begin{array}{l}\text { Influenza } \\
\text { (saisonal) }^{d}\end{array}$ & $\begin{array}{l}\text { Immer (ab einem } \\
\text { Alter von } 6 \text { Monaten } \\
\text { möglich) }\end{array}$ & $\begin{array}{l}1 \text { oder } 2 \text { Impfdosen ( } 2 \text { Impfdosen im 4-Wo- } \\
\text { chen-Abstand bei erster Influenzaimpfung im } \\
\text { Leben des Impflings, sonst reicht eine Einzeldosis) }\end{array}$ \\
\hline HPV & 9-17 Jahre & $\begin{array}{l}2 \text { oder } 3 \text { Impfdosen (0-1-6 Monate) }{ }^{e} \text { (siehe STIKO- } \\
\text { Empfehlungen [14]) }\end{array}$ \\
\hline
\end{tabular}

an Gemeinschaftseinrichtungen bevorzugt mit Hepatitis A kombinieren.

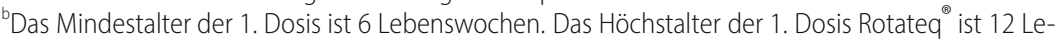
benswochen (keine Altersangabe für Rotarix ${ }^{\circledR}$ ). Das Höchstalter bei letzter Dosis ist 24 Lebenswochen (bei Rotarix ${ }^{\circledR}$ ) bzw. 32 Lebenswochen (bei Rotateq ${ }^{\circledR}$ ).

Vorzugsweise als MenACWY-Impfung.

${ }^{d}$ Der lebend-attenuierte Influenza-Impfstoff (LAIV; intranasale Applikation) ist der bevorzugte Impfstoff im Kindesalter und kann bei Kindern im Alter von 2-17 Jahren verwendet werden. Nach den STIKO-Empfehlungen ist er bevorzugt im Alter von 2-6 Jahren einzusetzen [14]. Für Säuglinge und Kinder unter 2 Jahre können nur parenterale inaktivierte Influenza-Totimpfstoffe (TIV) verwendet werden.

${ }^{\text {e}}$ Bei Immunisierungsbeinn bis zum Alter von 13 Jahren (mit Gardasil ${ }^{\oplus}$ ) bzw. bis zum Alter von 14 Jahren (d. h. bis ein Tag vor dem 15. Geburtstag) (mit Cervarix) muss nur 2-malig im Abstand von 6 Monaten geimpft werden (dann Mindestabstand für Cervarix 5 Monate).

sprache zwischen pädiatrischen Infektiologen/Tropenmediziner und Jugendgynäkologen empfohlen),

- Normozytäre Anämie (u. a. zum Ausschluss einer chronischen Infektion),

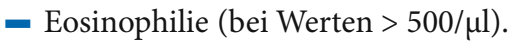

\section{Empfohlene Nachholimpfungen im Rahmen der ambulanten Versorgung}

Neben der ambulanten Versorgung von akuten oder chronischen Erkrankungen sollen im Rahmen der ambulanten Versorgung der Flüchtlinge (nach Verteilung der Flüchtlinge auf die Kommu- nen oder bei längeren Aufenthalten in Erstaufnahmeeinrichtungen) bestehende Impflücken geschlossen werden.

Bei den Nachholimpfungen gelten folgende Prinzipien [14]:

- Nur dokumentierte Impfungen sind applizierte Impfungen ${ }^{4}$.

- Jede dokumentierte Impfung zählt, egal wie lange sie her ist, das Immunsystem ,vergisst nicht“.

- Jede Nachimpfung soll in einem Impfausweis dokumentiert werden ${ }^{11}$. - Auffrischen oder Vervollständigen von Standardimpfungen, falls weniger Impfungen als von der STIKO aktuell empfohlen [14] dokumentiert sind und wenn der Patient die folgenden Alterskriterien erfüllt (- Tab.4).

Die STIKO hat seit dem Jahr 2012 Tabellen für verschiedene Altersgruppen publiziert, ${ }^{12}$ die als Anhaltspunkte für eine individuelle Planung von Nachholimpfungen zu verwenden sind und seitdem jährlich aktualisiert werden [14].

Da die meisten Flüchtlinge nach der Verteilung auf die Kommunen weiter in Gemeinschaftseinrichtungen (z. B. Asylbewerberheime) wohnen werden, ist dort die Infektionsgefährdung für Flüchtlinge höher als in der Allgemeinbevölkerung.

Der in dieser Stellungnahme empfohlene Impfkalender geht wegen der potenziell besseren medizinischen Versorgung in den Kommunen und dem längeren Aufenthalt der Flüchtlinge in den Gemeinschaftseinrichtungen (im Vergleich zu den Erstaufnahmestellen) über das Mindest-Impfangebot hinaus, dass das Robert Koch-Institut - in Abstimmung mit der STIKO - für Flüchtlinge in Erstaufnahmestellen veröffentlicht hat [16].

In dieser vorliegenden Stellungnahme werden Impfempfehlungen aufgrund der speziellen Lebenssituation in einer Gemeinschaftseinrichtung (in 0 Tab. 4 kursiv gedruckt) mit den Standardimpfempfehlungen der STIKO [14] kombiniert. Manche Impfzeitpunkte sind aufgrund der Dringlichkeit eines Immunschutzes bei Unterkunft in einer Gemeinschaftseinrichtung (in Analogie zu den STIKOEmpfehlungen für Erstaufnahmeeinrichtungen [16]) vorgezogen (in $\bullet \mathrm{Tab}^{5} \mathrm{mit}^{j}$ gekennzeichnet).

Müssen Flüchtlinge aus logistischen, administrativen oder politischen Gründen länger in Erstaufnahmeeinrichtungen verbleiben (> 4-6 Wochen), sollte auch diese Population sämtliche in - Tab. 5 angeführten Nachholimpfungen erhalten.

11 Ein Muster für ein Ersatzformular zur Dokumentation durchgeführter Impfungen (bei fehlendem Impfpass) findet sich in der STIKOPublikation [16].

12 Abzurufen unter http://www.rki.de/DE/ Content/Infekt/EpidBull/Archiv/2012/Ausgaben/30_12.pdf?_blob=publicationFile. 


\section{Konsensuspapiere}

Tab. 5 Empfohlener Impfkalender für Flüchtlinge im Kindes- und Jugendalter in Gemeinschaftseinrichtungen (nach Verteilung auf die Kommunen, gültig für Flüchtlinge, die das Stadium des Aufenthaltes in Erstaufnahmestellen bereits hinter sich haben, oder bei längeren Aufenthalten in Erstaufnahmeeinrichtungen)

\begin{tabular}{|c|c|c|}
\hline Impfungen & Kombination mit Vorsorgeuntersuchung & Vgl. zu STIKO-Empfehlungen [14] \\
\hline 2 Monate: DTaP-IPV-HepB-Hib + PCV + RV ${ }^{a}$ & & Dito \\
\hline 3 Monate: DTaP-IPV-HepB-Hib + $\left(\mathrm{PCV}^{\mathrm{b}}\right)+\mathrm{RV}$ & Bei U4 & Dito \\
\hline 4 Monate: DTaP-IPV-HepB-Hib + PCV + (RV) & & Dito \\
\hline 9 Monate: MMR $+V^{j}+T I V^{d}$ (Impfung ab 6 Monate möglich) & Spätestens bei U6 & Nein (ab 12 Monate für MMR + V) \\
\hline 12 Monate: MMRV + MenC oder MenACWY + HAV & Bei U6 & Nein (für MenACWY) \\
\hline 13 Monate: DTaP-IPV-HepB-Hib + PCV & & Dito (11-14 Mo) \\
\hline 15-23 Monate: evtl. Nachholimpfungen $+M M R V^{g}+H A V^{h}$ & Bei U7 & MMRV \\
\hline ab 24 Monate: $L A I V^{d}$ & & Nein \\
\hline 5-6 Jahre: Tdap & Bei U9 & Dito \\
\hline 9-17 Jahre: Tdap-IPV & & Dito \\
\hline 9-14 Jahre: HPV' für Mädchen & Spätestens bei J1 & Dito \\
\hline
\end{tabular}

${ }^{a}$ Rotavirus(RV)-Infektionsausbrüche in Flüchtlingslagern werden beschrieben, unklar, ob relevant unter der derzeitigen Situation in den deutschen Gemeinschaftsunterkünften.

bPneumokokken-Konjugat-Impfung (PCV) nur für Frühgeborene; bei Reifgeborenen 2+1-Schema.

'Bei Rotarix ${ }^{\circledast}$ zwei Impfdosen, bei Rotateq ${ }^{\oplus}$ drei Impfdosen. Zulassung nur für das Alter von 6 bis 24 Wochen (Rotarix ${ }^{\circledR}$ ) bzw. 6 bis 32 Wochen (Rotateq ${ }^{\circledR}$ ).

${ }^{d}$ Im Alter von 6 Monaten bis 8 Jahren sollen Kinder, die noch nie eine Influenza-Impfung (IV) erhalten haben, zwei Impfdosen im Abstand von mindestens 4 Wochen erhalten (TIV tri- oder tetravalente inaktivierte Influenza-Impfstoffe zur parenteralen Applikation; LAIV lebend-attenuierte Influenza-Impfstoffe zur intranasalen Applikation).

${ }^{e}$ Der Preis der tetravalenten MenACWY-Konjugatimpfstoffe ist vergleichbar mit dem der monovalenten MenC-Impfstoffe.

'Bei Aufenthalt in Gemeinschaftseinrichtungen empfohlen.

${ }^{9}$ Bei Erstimpfung mit MMRV vor dem 12. Lebensmonat und Zweitimpfung nach 4-6 Wochen ist eine 3. MMRV-Impfung notwendig (Mindestabstand zur 2. MMRV-Impfung von 3 Monaten)

${ }^{\mathrm{h}}$ Abstand zur ersten HAV-Impfung: 6-12 Monate.

'Impfschema: 0-6 Monate für Mädchen im Alter von 9 bis 13 Jahren (Gardasil ${ }^{\circledR}$ ) bzw. im Alter von 9 bis 14 Jahren (Cervarix ), 0-1(-2)-6 Monate für Mädchen ab einem Alter von 14 Jahren (Gardasi ${ }^{\circledR}$ ) bzw. 15 Jahren (Cervarix ${ }^{\circledR}$ ). Bis zum 18. Lebensjahr nachholen, falls vorher keine HPV-Impfung erfolgt ist.

'vorgezogen

Abstriche, die dem Nachweis einer Besiedelung mit multiresistenten Erregern (MRE) dienen (sogenanntes Kolonisationsscreening) sind bei Flüchtlingen und Asylbewerbern im Kindes- und Jugendalter im ambulanten Setting routinemäßig nicht empfohlen.

Anstelle eines routinemäßigen MREScreenings ist im ambulanten Bereich die sorgfältige Beachtung der Basishygiene [12] zielführend für den Umgang mit allen Patienten. Bei bekannter Besiedelung mit MRE sind spezielle Hygienemaßnahmen im ambulanten wie im stationären Setting zu ergreifen [8].

Die Frage der Notwendigkeit einer Dekolonisierung bei Nachweis einer Besiedelung mit MRSA bei einem ansonsten gesunden Kind ist Gegenstand einer sorgfältigen individualmedizinischen Risikoanalyse des behandelnden Kinder- und Jugendarztes. Im Falle einer MRSA-Infektion sollte parallel zur Behandlung eine Dekolonisation des Kindes angestrebt werden.

\section{Stationäre Vorstellung in den Kinderkliniken}

Die Vorstellung in einer Kinderklinik wird meist bei akuten Erkrankungen erfolgen. Neben dem symptomorientierten individuellen Untersuchungsprogramm soll die Vorstellung in einer Kinderklinik genutzt werden, um eventuell noch fehlende Untersuchungen aus dem Umfang der Basisuntersuchung zu ergänzen. Da$\mathrm{zu}$ ist entweder eine Dokumentation der Basisuntersuchung notwendig (z. B. Einlegeblatt mit den Ergebnissen der Basisuntersuchung im U-Heft oder Impfausweis) oder eine Kommunikationsstruktur mit der lokalen Erstaufnahmestelle bzw. den lokalen Praxisärzten und -ärztinnen notwendig. Eine solche Struktur existiert derzeit nicht und sollte aufgebaut werden.

Zusätzlich zur eventuellen Komplettierung der Basisuntersuchung soll bei Flüchtlingen ein MRE-Screening vor/bei jeder stationären Aufnahme erfolgen, wenn der Patient

1. innerhalb der letzten 12 Monate im Herkunftsland bzw. im Transit einen Krankenhausaufenthalt oder wiederholten Kontakt mit Einrichtungen des Gesundheitssystem hatte oder

2. eine bekannte frühere Kolonisierung oder Infektion mit MRE hatte oder

3. chronische Wunden/Hautläsionen aufweist.

Darüber hinaus sollte ein MRE-Screening erfolgen, wenn der Patient in der Klinik Kontakte zu potenziell gefährdeten Risikopatienten, z.B. aus den Bereichen Intensivtherapie, Onkologie oder Transplantation hat und

1. eine Flüchtlingsanamnese in den letzten 3 Monaten ${ }^{13}$ hatte oder

${ }^{13}$ Die Grenze von 3 Monaten ist arbiträr gewählt, da es keine Daten zur MRE-Besiedelung bei Flüchtlingen gibt. Bei neuen Erkenntnissen muss die Zeitangabe ggf. revidiert werden. 
2. in einer Gemeinschaftseinrichtung untergebracht ist.

Zum Screening benötigte Proben werden von folgenden Körperstellen abgenommen:

- beide Nasenvorhöfe und Rachen (MRSA) - 2 Tupfer,

- beide Leisten (MRSA) - ein Tupfer für beide Stellen ausreichend,

- Rektalabstrich (multiresistente gramnegative Bakterien; MRGN) - ein Tupfer ausreichend, Durchtritt durch den Analsphinkter notwendig!

Bis zum Erhalt der MRE-Screeningergebnisse ist bei stationären Patienten eine prophylaktische Isolierung empfohlen (soweit dies die baulichen Bedingungen zulassen).

\section{Untersuchungsprogramm für schwangere Flüchtlinge bei Erstvorstellung}

- Frage nach Husten, Fieber, Gewichtsverlust, Nachtschweiß,

- Impfstatus erheben,

- Messung von Körpertemperatur, Atemfrequenz, Herzfrequenz, Blutdruck, Körpergewicht,

- körperliche Untersuchung von Lunge, Abdomen (inkl. Nieren).

Schwangere sollen danach bzw. nach Feststellung einer Schwangerschaft einer niedergelassenen Frauenärztin ${ }^{14}$ vorgestellt werden. Dort sind folgende zusätzliche Untersuchungen - nach den „Mutterschafts-Richtlinien“ - empfohlen:

- Urin-Status auf Eiweiß,

- kapilläres/venöses Blutbild zur Hämoglobin-Kontrolle,

- serologische Untersuchungen auf Hepatitis B (HBs-Antigen; idealerweise nach der 32. Schwangerschaftswoche, möglichst nahe am errechneten Geburtstermin), HIV (anti-HIV; nach entsprechender Aufklärung), Lues (TPPA/TPHA/ELISA), Röteln (falls keine zwei Röteln-Impfungen erfolgt sind),

14 Aus kulturellen Gründen sollte eine Vorstellung bei einer Frauenärztin bevorzugt werden
- Ein Rektovaginalabstrich zwischen der 34. und 37. Schwangerschaftswoche auf Gruppe-B-Streptokokken und multiresistente gram-negative Erreger (MRGN) sollte aufgrund der zu erwartenden höheren Inzidenz einer Besiedelung in der Flüchtlingspopulation durchgeführt werden,

- Abstriche auf multiresistente Erreger (MRE) sind bei schwangeren Flüchtlingen im Jugendalter im ambulanten Setting routinemäßig nicht zu fordern. Die Testung macht jedoch Sinn bei allen Schwangeren in der Spätschwangerschaft (z. B. mit einem GBS-Screening kombiniert), spätestens bei stationärer Aufnahme zur Geburt oder bei vorzeitiger Wehentätigkeit,

- Influenza-Impfung mit Influenza-

Totimpfstoffen sind zu empfehlen ( $\mathrm{ab}$ der 20. Schwangerschaftswoche),

- bei negativer Röteln-Serologie Aufklärung über Verhalten bei RötelnExposition und aktive Röteln-Impfung nach der Entbindung empfehlen; dann kombiniert als MMR-Impfung (ggf. zusätzliche Varizellenimpfung),

- Totimpfstoffe wie Tdap-Impfstoffe können in der Schwangerschaft gegeben werden, sodass bestehende Impflücken ggf. auch in der Schwangerschaft geschlossen werden können. Impfungen sollten jedoch nicht vor dem 2. Trimenon durchgeführt werden.

\section{Finanzierungsaspekte}

Die Finanzierung von medizinischen Leistungen bei Flüchtlingen ist nach dem Asylbewerberleistungsgesetz (AsylbLG) geregelt. Flüchtlinge erhalten - über den Umweg einer Einzelbeantragung bei den Behörden - einen Krankenbehandlungsschein (oder Versichertenkarte - ja nach Bundesland) mit eingeschränktem Leistungsanspruch. In einigen Bundesländern (z. B. Hamburg, Bremen) wurde dieses Verfahren durch Gesundheitskarten ersetzt. Kosten werden erstattet für Behandlungen von

3. akut notwendigen, nicht aufschiebbaren Erkrankungen,

4. Schmerzen,

5. Mutterschaftsvorsorgeleistungen,
Hier steht eine Anzeige. Springer 
6. Früherkennungsuntersuchungen U1 bis U9,

7. Gesundheitsuntersuchung J1,

8. Schutzimpfungen.

Leistungen für vorbestehende chronische Erkrankungen müssen nicht vergütet werden. In diesen Fällen muss laut Gesetzeslage immer eine Kostenzusage vor der Behandlung eingeholt werden. Diese restriktive Haltung wurde vom 118. Deutschen Ärztetag in Frankfurt/M. kritisiert und als ethisch fragwürdig angesehen [3]. Die Einschränkung von medizinischen Leistungen ist auch ökonomisch nicht sinnvoll, da höhere Folgekosten damit verbunden sind [4]. So lagen in einer Studie die ProKopf-Ausgaben für die eingeschränkte medizinische Versorgung um ca. $40 \%$ höher als bei Asylsuchenden mit regulärem Anspruch auf kassenärztliche Leistungen [4]. Zusätzlich ist der Verwaltungsaufwand für Einzelbegutachtungen von Leistungsanträgen erheblich und verursacht zusätzliche Kosten für die zuständigen Ämter.

Kostenträger im Rahmen des Asylbewerberleistungsgesetzes ist die jeweilige Kommune, in Einzelfällen das Bundesland. Die Abwicklung erfolgt über das örtlich zuständige Sozialamt.

Nach Gewährung von Asyl oder nach 15 Monaten Aufenthalt in Deutschland - bei noch nicht abgeschlossenem Asylverfahren - hat der Asylbewerber einen Anspruch auf eine Vollversicherung über eine gesetzliche Krankenkasse. Einige bundesdeutsche Länder (z. B. Hamburg und Bremen) stellen bereits nach Antrag auf Asyl eine Gesundheitskarte aus, über die Asylbewerber bei einer gesetzlichen Krankenversicherung mit den vorgenannten Einschränkungen versichert sind und sich direkt bei einem Arzt mit Kassenzulassung vorstellen können. Nordrhein-Westfalen hat im August 2015 mit Krankenkassen eine Rahmenvereinbarung zur Einführung der Gesundheitskarte beschlossen, in einigen Ländern ist dies geplant. ${ }^{15}$ Die Delegierten des 118. Deutschen Ärztetag in Frankfurt/M. fordern für alle Flüchtlinge in Deutschland die Einführung einer Versichertenkarte [3].
Im Gegensatz zu Kindern, die gemeinsam mit ihren Eltern nach Deutschland flüchten und nach dem AsylbLG einen eingeschränkten Leistungsanspruch auf medizinische Versorgung haben, erhalten unbegleitete minderjährige Flüchtlinge (UMF), die in die Obhut des Jugendamtes genommen werden, ab diesem Moment die Leistungen der gesetzlichen Krankenversicherung.

Die Versorgung der Flüchtlinge insbesondere im stationären Bereich mit schweren Erkrankungen, eingreifenden Therapien einschließlich Operationen und unterschiedlicher Prognose erfordert erhebliche zusätzliche Ressourcen. Es müssen Dolmetscher gefunden und bezahlt werden. Alle Untersuchungen, Aufklärungen, Therapien und Visiten dauern meist ein Mehrfaches der bei deutschen Patienten aufzuwendenden Zeit. Die problematische Unterkunft in den Heimen macht es notwendig, Kinder aufzunehmen, die bei Vorhandensein einer intakten Wohnung zu Hause gepflegt werden könnten. Kinder können aus dem gleichen Grund nicht nach Hause entlassen werden, wenn eine häusliche Nachbehandlung nicht gewährleistet ist. Deshalb müssen zusätzliche Mittel für die Versorgung dieser Kinder bereitgestellt werden, z. B. in Form einer Zusatz-DRG, deren Wert auszuhandeln ist.

Die Delegierten des 118. Deutschen Ärztetag in Frankfurt/M. beschlossen: „Alle Flüchtlinge müssen vollen $\mathrm{Zu}$ gang $\mathrm{zu}$ allen Gesundheitsleistungen der gesetzlichen Krankenkassen bekommen" [3]. Die Delegierten berufen sich dabei auf die UN-Kinderrechtskonvention (Art. 24, Abs. 1), die „das erreichbare Höchstmaß an Gesundheit“ als ein Grundrecht jedes Kindes nennt. Neben dem Zugang zu optimaler gesundheitlicher Versorgung fordert die UN-Kinderrechtskonvention auch einen Zugang zu Bildung und $\mathrm{zu}$ sozialer Teilhabe aller Flüchtlingskinder. Die

\footnotetext{
15 Der Stand zur Einführung von Gesundheitskarten für Flüchtlinge in den einzelnen Bundesländern kann hier nachgelesen werden: www.aerztezeitung.de/ politik_gesellschaft/gp_specials/fluechtlinge/ default.aspx?sid $=896098 \& \mathrm{~cm} \_m m c=$ Newsletter-_-Newsletter-C__-20151013-_-Flüchtling.
}

Autoren der Stellungnahme unterstützen die Forderung nach Einführung einer elektronischen Gesundheitskarte und Zugang zu Leistungen der gesetzlichen Krankenversicherung für alle Flüchtlinge in Deutschland.

\section{Fazit für die Praxis}

- Die Sicherstellung einer adäquaten medizinischen Versorgung von Flüchtlingen im Kindes- und Jugendalter stellt eine große Herausforderung dar.

- Bei Kindern und Jugendlichen soll frühzeitig nach Ankunft in einer Erstaufnahmestelle eine ärztliche Basisuntersuchung mit fokussierter Anamnese, klinischer Untersuchung und Erfassung des Impfstatus erfolgen.

- Ein generelles Tuberkulosescreening (mit Tuberkulin-Hauttest [0-14 Jahre] oder Interferon-gamma Release Assay [5-14 Jahre]) ist bei allen Flüchtlingen im Kindes- und Jugendalter in den Erstaufnahmestellen empfohlen, wird dort aber erfahrungsgemäß personell und organisatorisch an seine Grenzen stoßen. Ist ein generelles Tuberkulosescreening nicht umsetzbar, soll zunächst ein risikobasiertes Tuberkulosescreening erfolgen. Das Screeningergebnis bzw. ein nichtdurchgeführtes Screening ist entsprechend zu dokumentieren.

- Bestehende Impflücken sollen in der Erstaufnahmestelle sobald wie möglich geschlossen werden. Dabei haben Impfungen gegen Masern, Mumps, Röteln, Varizellen die höchste Priorität, gefolgt von Diphtherie, Tetanus, Pertussis, Poliomyelitis, sowie saisonale Influenza.

- Im Rahmen einer ambulanten Weiterbehandlung ist die Bestimmung eines Differenzialblutbildes, bei Herkunft aus Hochprävalenzländern (nicht Syrien) auch eine serologische Untersuchung auf HIV und Hepatitis B empfohlen.

- Bei der stationären Aufnahme in Kinderkliniken sollte ein Screening auf multiresistente Erreger durchgeführt werden, insbesondere wenn 


\section{Hinweise auf einen früheren Kran- kenhausaufenthalt bestehen. - Unabdingbar, und derzeit vielerorts unzureichend, ist die Dokumentation und Weitergabe medizinischer Be- funde an den Schnittstellen zwischen Erstaufnahmestelle sowie ambulan- ter und stationärer Versorgung.}

\section{Korrespondenzadresse

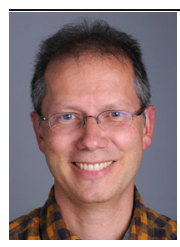 \\ PD Dr. M. Hufnagel \\ Sektion Pädiatrische Infektiologie und Rheumatologie, Klinik I, Zentrum für Kinder- und Jugendmedizin, Universitätsklinikum Freiburg Mathildenstr. 1, 79106 Frei- burg, Deutschland markus.hufnagel@uniklinik- freiburg.de}

Danksagung. Die Autoren danken zusätzlich folgenden Kollegen und Kolleginnen, die mit Ihren Kommentaren bzw. Beiträgen an der Fertigstellung der Stellungnahme beigetragen haben: Dr. Monika Engel (Kinder- und Jugendärztin aus Karlsruhe), Dr. Roland Fressle (Kinder- und Jugendarzt aus Freiburg, Landesverbandsvorsitzender des BVKJ BadenWürttemberg), Prof. Dr. Walter Haas (Fachgebiet „Respiratorisch übertragbare Erkrankungen“ der Abteilung für Infektionsepidemiologie am Robert Koch-Institut; Ausschuss typische und atypische Mykobakteriosen der DGPI), Dr. Ulrike Horacek, MPH (Gesundheitsamt Recklinghausen), Dr. Axel Iseke, MPH (Gesundheitsamt Münster), Dr. Martina Sappa und Dr. Torsten Spranger (Autoren des „Bremer Gesundheitsheftes"), sowie Dr. Miriam Wiese-Posselt, MPH (Robert Koch-Institut - Fachgebiet Impfprävention).

\section{Einhaltung ethischer Richtlinien}

Interessenkonflikt. J. Pfeil, R. Kobbe, S. Trapp, C. Kitz und M. Hufnagel geben an, dass kein Interessenkonflikt besteht.

Dieser Beitrag beinhaltet keine Studien an Menschen oder Tieren.

\section{Literatur}

1. American Academy of Pediatrics (2012) Comprehensive health evaluation of the newly adopted child. Pediatrics 129:e214-e223

2. Ritz N, Brinkmann F, Feiterna-Sperling $C$ et al Arbeitsgruppe AWMF-Leitlinie Diagnostik, Prävention und Therapie der Tuberkulose im Kindesund Jugendalter unter Federführung der Deutschen Gesellschaft für Pädiatrische Infektiologie. Tuberkulosescreening bei asylsuchenden Kindern und Jugendlichen $<15$ Jahren in Deutschland. Stellungnahme der Arbeitsgruppe AWMFLeitlinie Tuberkulose im Kindes- und Jugendal- ter: Diagnostik, Prävention und Therapie unter Federführung der Deutschen Gesellschaft für Pädiatrische Infektiologie. Monatsschr Kinderheilkd 163. doi:10.1007/s00112-015-0007-5

3. Bundesärztekammer (2015) Beschlussprotokoll des 118. Deutschen Ärztetages in Frankfurt am Main vom 12. bis 15.05.2015. http:// www.bundesaerztekammer.de/aerztetag/118 deutscher-aerztetag-2015/beschlussprotokoll/. Zugegriffen:22.11.2015

4. Bozorgmehr K, Razum O (2013) Effect of restricting access to health care on health expenditures among asylum-seekers and refugees: A quasi-experimental study in Germany, 1994-2013. PLoS One 10(7):e0131483 doi:10.1371/journal.pone.0131483

5. Bundesamt für Migration und Flüchtlinge (2015) Sehr hoher Asyl-Zugang im September (Stand 07.10.2015). http://www. bamf.de/SharedDocs/Meldungen/DE/2015/ 20151007-asylgeschaeftsstatistik-september. html?nn=1367522.Zugegriffen:22.11.2015

6. Deutsche Gesellschaft für Pädiatrische Infektiologie (2013) DGPI-Handbuch - Infektionen bei Kindern und Jugendlichen, 6 . Aufl. Thieme, Stuttgart

7. Deutsche Gesellschaft für Sozialpädiatrie und Jugendmedizin (2015) Pressegespräch zur 110. Jahrestagung der Deutschen Gesellschaft für Kinder- und Jugendmedizin vom 1. September 2015. Medizinische Versorgung minderjähriger Flüchtlinge in Deutschland: Eine neue Herausforderung für die Kinder- und Jugendmedizin. http://www. dgkj.de/fileadmin/user_upload/images/Presse/ Jahrestagung_2015/1509_DGSPJ.pdf. Zugegriffen:22.11.2015

8. Arbeitsgruppe MRGN der Deutschen Gesellschaft für Pädiatrische Infektologie (2015) Empfehlung der Arbeitsgruppe MRGN der Deuschen Gesellschaft für Pädiatrische Infektiologie und des Paed IC Projekts zum Infektionspräventivem Vorgehen bei Nachweis von MRGN im Kindesalter. http://dgpi.de/umgang-mit-mrgn-bei-kindern. Zugegriffen: 22.11.2015

9. Eurostat (2015) Pressemitteilung 163/2015 vom 18. September 2015. Asyl in der EU. Über 210000 erstmalige Asylbewerber in der EU im zweiten Quartal 2015. http://ec.europa. eu/eurostat/documents/2995521/6996930/318092015-BP-DE.pdf/d08b4652-2b94-4da09fff-bb2924a09754.Zugegriffen:22.11.2015

10. Graham SM, Cuevas LE, Jean-Philipp $P$ et al (2015) Clinical case definitions for classification of intrathoracic tuberculosis in children: An update. Clin Infect Dis 61(Suppl3):S179-S187

11. Marburger Bund Nordrhein-Westfalen-RheinlandPfalz (2015) Hauptversammlung des Marburger Bundes Nordrhein-Westfalen-Rheinland-Pfalz in Köln vom 23.09.2015, Kölner Statement zur medizinischen Versorgung von Flüchtlingen. https:// www.marburger-bund.de/landesverbaende/ nrw-rlp/artikel/allgemein-hauptversammlung/ 2015/klinikfinanzierung-muss-zukunftssichergestaltet-werden.Zugegriffen:22.11.2015

12. Heudorf U, Hausemann A, Hofmann $\mathrm{H}$ et al (2013) Hygiene und Infektionsprävention in der Kinder- und Jugendarztpraxis - Anforderungen und Beobachtungen. Monatsschr Kinderheilkd 161:925-934

13. Kimbrough W, Saliba V, Dahab M et al (2012) The burden of tuberculosis in crisis-affected populations: a systematic review. Lancet Infect Dis 12:950-965
14. STIKO (Ständige Impfkommission am Robert-Koch-Institut) (2015) Mitteilungen der Ständigen Impfkommission (STIKO) am Robert Koch-Institut / August 2015. Epidemiol Bull 34:327-362 (https://www.rki.de/DE/ Content/Infekt/EpidBull/Archiv/2015/Ausgaben/ 34_15.pdf?_blob=publicationFile). Zugegriffen: 22.11.2015

15. Robert-Koch-Institut (2015) Für medizinisches Personal: Akut behandlungsbedürftige, für Deutschland ungewöhnliche Infektionskrankheiten, die bei Asylsuchenden auftreten können. Epidemiol Bull 38:413-414 (http://edoc.rki.de/oa/ articles/reKBnmZGiO1Vs/PDF/290AiZ9cxqll.pdf). Zugegriffen:22.11.2015

16. Robert-Koch-Institut (2015) Konzept zur Umsetzung frühzeitiger Impfungen bei Asylsuchenden nach Ankunft in Deutschland. Epidemiol Bull 41:439-444 (http://edoc.rki.de/oa/articles/ re1ySqxtCvl/PDF/28OLguEy24AYY.pdf). Zugegriffen: 22.11.2015

17. Robert Koch-Institut (2015) Infektionskrankheiten A bis Z. http://www.rki.de/DE/Content/ InfAZ/InfAZ_marginal_node.html. Zugegriffen: 22.11.2015

18. Robert-Koch-Institut (2015) Thorax-Röntgenuntersuchungen bei Asylsuchenden gemäß $\$ 36$ Absatz 4 IfSG. http://www.rki.de/DE/Content/ InfAZ/T/Tuberkulose/Tuberkulose_RoentgenUntersuchungen_Asylsuchende.html (Erstellt: 5.10.2015).Zugegriffen: 22.11.2015

19. DAKJ e. V. (Deutsche Ärztekommission für Kinder- und Jugendmedizin (2014) Stellungnahme der Kommission für Infektionskrankheiten und Impffragen der Deutschen Akademie für Kinderund Jugendmedizin (DAKJ e.V.) zu Medizinischen Maßnahmen bei immigrierenden Kindern und Jugendlichen vom Februar 2008; Aktualisierung vom 07.10.2013. Monatsschr Kinderheilkd 162:56-61

20. Swanson SJ, Phares CR, Mamo B et al (2012) Albendazole therapy and enteric parasites in United States-bound refugees. N Engl J Med 366:1498-1507

21. Tamashiro VG, Perez HH, Griffin DE (1987) Prospective study of the magnitude and duration of changes in tuberculin reactivity during uncomplicated and complicated measles. Pediatr Infect Dis $6: 451-454$

22. World Health Organisation (2002) Hepatitis B. www.who.int/csr/disease/hepatitis/ whocdscsrlyo20022/en/index3.html. Zugegriffen: 22.11.2015

23. World Health Organization (2013) HIV/AIDS - Definition of key terms. http://www.who.int/hiv/pub/ guidelines/arv2013/intro/keyterms/en/ (Erstellt: Jun 2013).Zugegriffen: 22.11.2015

24. World Health Organization (2015) Tuberculosis country profiles. http://www.who.int/tb/country/ data/profiles/en/.Zugegriffen: 22.11.2015

25. U.S. Department of Health and Human Services, Centers for Disease Control and Prevention, National Center for Emerging and Zoonotic Infectious Diseases (2012) General Refugee Health Guidelines vom 6. August 2012. http://www. cdc.gov/immigrantrefugeehealth/guidelines/ general-guidelines.html\#chemistries. Zugegriffen: 22.11 .2015 
Hier steht eine Anzeige.

算 Springer 